\title{
CARMA observations of Galactic cold cores: searching for spinning dust emission
}

\author{
C. T. Tibbs, ${ }^{1,2 \star} \dagger$ R. Paladini, ${ }^{2}$ K. Cleary, ${ }^{3}$ S. J. C. Muchovej, ${ }^{3}$ A. M. M. Scaife, ${ }^{4}$ \\ M. A. Stevenson, ${ }^{3}$ R. J. Laureijs, ${ }^{1}$ N. Ysard,${ }^{5}$ K. J. B. Grainge, ${ }^{4}$ Y. C. Perrott, ${ }^{6}$ \\ C. Rumsey ${ }^{6}$ and J. Villadsen ${ }^{3}$ \\ ${ }^{1}$ Scientific Support Office, Directorate of Science and Robotic Exploration, European Space Research and Technology Centre (ESA/ESTEC), \\ Keplerlaan 1, NL-2201 AZ Noordwijk, the Netherlands \\ ${ }^{2}$ Infrared Processing Analysis Center, California Institute of Technology, Pasadena, CA 91125, USA \\ ${ }^{3}$ Cahill Center for Astronomy and Astrophysics, California Institute of Technology, Pasadena, CA 91125, USA \\ ${ }^{4}$ Jodrell Bank Centre for Astrophysics, The University of Manchester, Manchester M13 9PL, UK \\ ${ }^{5}$ IAS, Université Paris-Sud, F-91405 Orsay cedex, France \\ ${ }^{6}$ Astrophysics Group, Cavendish Laboratory, University of Cambridge, Cambridge CB3 OHE, UK
}

Accepted 2015 July 30. Received 2015 July 30; in original form 2015 March 30

\begin{abstract}
We present the first search for spinning dust emission from a sample of 34 Galactic cold cores, performed using the CARMA interferometer. For each of our cores, we use photometric data from the Herschel Space Observatory to constrain $\bar{N}_{\mathrm{H}}, \bar{T}_{\mathrm{d}}, \bar{n}_{\mathrm{H}}$, and $\bar{G}_{0}$. By computing the mass of the cores and comparing it to the Bonnor-Ebert mass, we determined that 29 of the 34 cores are gravitationally unstable and undergoing collapse. In fact, we found that six cores are associated with at least one young stellar object, suggestive of their protostellar nature. By investigating the physical conditions within each core, we can shed light on the $\mathrm{cm}$ emission revealed (or not) by our CARMA observations. Indeed, we find that only three of our cores have any significant detectable $\mathrm{cm}$ emission. Using a spinning dust model, we predict the expected level of spinning dust emission in each core and find that for all 34 cores, the predicted level of emission is larger than the observed $\mathrm{cm}$ emission constrained by the CARMA observations. Moreover, even in the cores for which we do detect $\mathrm{cm}$ emission, we cannot, at this stage, discriminate between free-free emission from young stellar objects and spinning dust emission. We emphasize that although the CARMA observations described in this analysis place important constraints on the presence of spinning dust in cold, dense environments, the source sample targeted by these observations is not statistically representative of the entire population of Galactic cores.
\end{abstract}

Key words: ISM: clouds - dust, extinction - ISM: evolution - ISM: general-infrared: ISM radio continuum: ISM.

\section{INTRODUCTION}

In recent years, there has been mounting evidence for the existence of a new emission component of the Galactic interstellar medium (ISM). This new component was first identified in the diffuse ISM, and, due to its unexpected discovery, was simply referred to as anomalous microwave emission (Leitch et al. 1997). We now believe that this anomalous microwave emission is due to electric dipole

^E-mail: ctibbs@cosmos.esa.int

†ESA Research Fellow. emission from small interstellar dust grains, commonly known as spinning dust emission (Draine \& Lazarian 1998).

Spinning dust emission occurs in the wavelength range $\sim 3-$ $0.3 \mathrm{~cm}$ and produces a very distinctive peaked spectrum, peaking at wavelengths of $\sim 1 \mathrm{~cm}$, with the exact wavelength depending on the physical environmental conditions. Although this microwave emission from small spinning dust grains has been found in a wide variety of environments, the best examples of spinning dust detections occur in dense environments: the Perseus molecular cloud (Watson et al. 2005; Tibbs et al. 2010, 2013; Planck Collaboration XX 2011; Génova-Santos et al. 2015), and the dark cloud LDN1622 (Finkbeiner 2004; Casassus et al. 2006; Harper, Dickinson \& Cleary 2015). In this analysis we attempt, for the first time, to search for 
Table 1. Summary of the 15 cold clumps targeted in this analysis.

\begin{tabular}{lcccrcc}
\hline & & & & \multicolumn{3}{c}{ CARMA rms } \\
Target & $\begin{array}{c}\text { RA } \\
(\mathrm{J} 2000)\end{array}$ & $\begin{array}{c}\text { Dec. } \\
(\mathrm{J} 2000)\end{array}$ & $\begin{array}{c}\text { Distance } \\
(\mathrm{kpc})\end{array}$ & $\begin{array}{c}T_{\text {gas }} \\
(\mathrm{K})\end{array}$ & $\begin{array}{c}\sigma_{\text {Short }} \\
\left(\mathrm{mJy} \mathrm{beam}^{-1}\right)\end{array}$ & $\begin{array}{c}\sigma_{\text {Long }} \\
\left(\mathrm{mJy} \mathrm{beam}^{-1}\right)\end{array}$ \\
\hline ECC181 G102.19+15.24 & $20: 41: 10.74$ & $+67: 21: 44.3$ & $0.33^{a}$ & 9.6 & 0.58 & 0.55 \\
ECC189 G103.71+14.88 & $20: 53: 30.29$ & $+68: 19: 32.9$ & $0.29^{a}$ & 9.7 & 0.37 & 0.37 \\
ECC190 G103.77+13.90 & $21: 02: 09.19$ & $+67: 45: 51.8$ & $0.29^{a}$ & 11.1 & 0.45 & 0.48 \\
ECC191 G103.90+13.97 & $21: 02: 23.24$ & $+67: 54: 43.3$ & $0.29^{a}$ & 11.1 & 0.90 & 0.86 \\
ECC223 G113.42+16.97 & $21: 59: 59.03$ & $+76: 34: 08.7$ & $0.99^{b}$ & 8.9 & 0.49 & 0.47 \\
ECC224 G113.62+15.01 & $22: 21: 37.34$ & $+75: 06: 33.5$ & $0.86^{b}$ & 8.0 & 0.75 & 0.78 \\
ECC225 G113.75+14.90 & $22: 24: 16.23$ & $+75: 05: 01.8$ & $0.88^{b}$ & 8.6 & 0.67 & 0.61 \\
ECC229 G114.67+14.47 & $22: 39: 35.57$ & $+75: 11: 34.0$ & $0.77^{b}$ & 10.3 & 0.45 & 0.38 \\
ECC276 G127.88+02.66 & $01: 38: 39.14$ & $+65: 05: 06.5$ & $1.16^{b}$ & 12.6 & 0.33 & 0.37 \\
ECC332 G149.41+03.37 & $04: 17: 09.10$ & $+55: 17: 39.4$ & $0.18^{b}$ & 8.7 & 0.52 & 0.59 \\
ECC334 G149.58+03.45 & $04: 18: 23.96$ & $+55: 13: 30.6$ & $0.20^{b}$ & 8.7 & 0.52 & 0.59 \\
ECC335 G149.65+03.54 & $04: 19: 11.28$ & $+55: 14: 44.4$ & $0.17^{b}$ & 8.1 & 0.57 & 0.65 \\
ECC340 G151.45+03.95 & $04: 29: 56.29$ & $+54: 14: 51.7$ & $0.19^{a}$ & 10.1 & 0.36 & 0.39 \\
ECC345 G154.07+05.09 & $04: 47: 23.41$ & $+53: 03: 31.4$ & $0.34^{b}$ & 7.4 & 0.59 & 0.63 \\
ECC346 G154.07+05.21 & $04: 47: 57.83$ & $+53: 07: 51.2$ & $0.23^{b}$ & 10.0 & 0.67 & 0.64 \\
\hline
\end{tabular}

$\overline{{ }^{a} \text { Distances are based on association with known Lynds dark nebulae (Hilton \& Lahulla 1995). }{ }^{b} \text { Distances are }}$ kinematic distances based on ${ }^{13} \mathrm{CO}$ observations (Wu et al. 2012). $T_{\text {gas }}$ values taken from Wu et al. (2012).

spinning dust emission in even more dense environments: Galactic cores. Galactic cores represent one of the earliest phases of star formation, created by the gravitational collapse of an over density within a molecular cloud (see Bergin \& Tafalla 2007, for a complete review). Although no such observations of spinning dust emission from Galactic cores have been performed, Ysard, Juvela \& Verstraete (2011) conducted radiative transfer modelling of such dense cores and predicted that spinning dust emission should be detectable at microwave frequencies if there are small dust grains present. Additionally, Ysard et al. (2011) showed that as the density of the cores increases, an anticorrelation between the microwave emission and the mid-infrared (IR) emission occurs. The mid-IR emission is concentrated around the edge of the dense cores, while the microwave emission is contained within the centre of the cores. Ysard et al. (2011) proposed that this anticorrelation could be explained in two ways. Either the small grains that produce both the mid-IR emission and the microwave emission are present throughout the core, but due to the high densities, the stellar photons cannot penetrate deep enough into the core to cause the small grains to emit at mid-IR wavelengths, or that there is a deficit of small grains in the centre. Therefore, the motivation for this current work was to search for spinning dust emission in dense cores, and determine which of these two hypotheses is correct. To this end, we observed a sample of Galactic cores with the CARMA interferometer at a wavelength of $1 \mathrm{~cm}$, which based on the work by Ysard et al. (2011), is the expected peak wavelength of the spinning dust emission in these dense environments.

This paper is organized as follows. In Section 2, we define our sample, and in Section 3, we describe the data that we use to perform this analysis. In Section 4, we characterize the physical properties of our cores, and in Section 5, we compare our observations with the expected level of spinning dust emission. Finally, we present our conclusions in Section 6.

\section{SOURCE SELECTION}

For the dust temperatures characteristic of Galactic cold cores $(\sim 8-14 \mathrm{~K})$, the peak of the thermal dust emission occurs around $\sim 200 \mu \mathrm{m}$, implying that the best wavelength to detect these objects is in the far-IR and sub-mm bands. Planck, with its all-sky coverage at sub-mm wavelengths, is an ideal instrument with which to detect cold cores. In fact, as part of the Planck Early Release Compact Source Catalogue (Planck Collaboration VII 2011), the Early Compact Core (ECC) catalogue was published. The ECC catalogue contains 915 cold clumps ${ }^{1}$ identified by the Planck team to be the coldest $(T<14 \mathrm{~K})$ and most reliable $(\mathrm{SNR}>15)$ sources in the Cold Core Catalogue of Planck Objects (C3PO).

We selected a sample of 15 sources from the ECC catalogue. The sources were selected based on the following criteria: (a) the source must have been observed with the Herschel Space Observatory PACS and SPIRE photometers (Pilbratt et al. 2010); (b) the source must be visible from the site of the CARMA interferometer; (c) the source must have an angular size less than 12 arcmin to ensure that we do not resolve out any flux; (d) the source must have a known distance estimate; and (e) the sources must cover a range of densities, $n_{\mathrm{H}}$. The 15 sources included in our sample are listed in Table 1.

Also listed in Table 1 are the distance estimates for each source. Wu et al. (2012) observed the $J=1-0$ transition of ${ }^{12} \mathrm{CO},{ }^{13} \mathrm{CO}$, and $\mathrm{C}^{18} \mathrm{O}$ for 674 of the $915 \mathrm{ECC}$ sources using the $13.7 \mathrm{~m}$ telescope of the Purple Mountain Observatory. Using the ${ }^{13} \mathrm{CO} V_{\mathrm{LSR}}$ measurements with the Clemens (1985) rotation curve, and adopting values of $R_{0}=8.5 \mathrm{kpc}$ and $\Theta_{0}=220 \mathrm{~km} \mathrm{~s}^{-1}$, Wu et al. (2012) computed the kinematic distance to each source. For sources within the solar circle, which suffer from the kinematic distance ambiguity, they selected the near distance. Since Wu et al. (2012) were unable to assign a distance estimate to all of their ECC sources, there were five sources in our sample (ECC181, ECC189, ECC190, ECC191, and ECC340) for which we did not have a kinematic distance estimate, and for these sources we estimated the distance based on associations with known Lynds dark nebulae (Hilton \& Lahulla 1995).

\footnotetext{
${ }^{1}$ Since the angular resolution of Planck is $\sim 5$ arcmin in the far-IR/sub$\mathrm{mm}$ bands, it cannot detect individual cores, and therefore we refer to the Planck sources as clumps.
} 


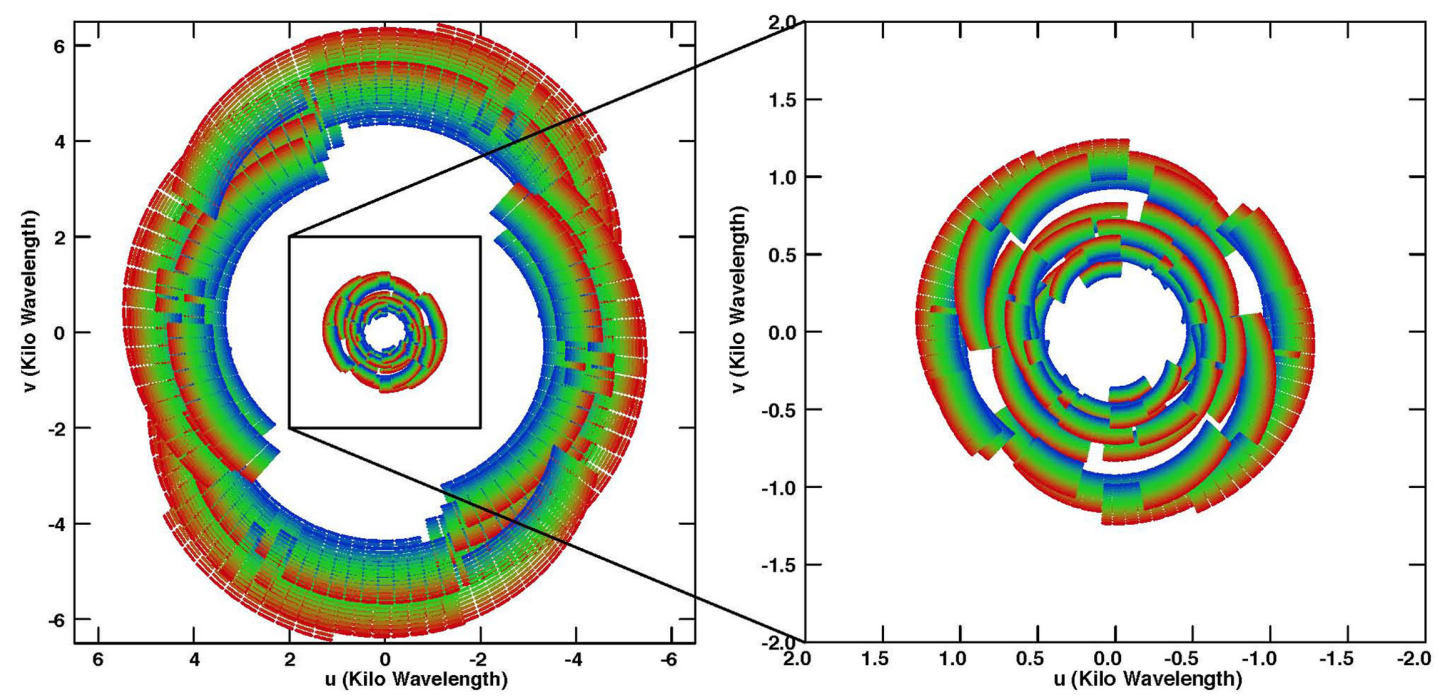

Figure 1. Complete $u-v$ coverage (left) and the $u-v$ coverage for only the short baselines (right) for our observations of ECC181. The colour scale represents the 15 channels with red corresponding to the highest frequency channel and blue corresponding to the lowest frequency channel. The $u$ - $v$ coverage for the rest of our targets is very similar.

In addition to a distance estimate, Wu et al. (2012) also derived the temperature of the gas, $T_{\text {gas }}$, for all of their ECC sources. The $T_{\text {gas }}$ values for our 15 targets are also listed in Table 1, and these values are adopted throughout this analysis.

\section{OBSERVATIONS}

\subsection{CARMA data}

CARMA is an interferometer consisting of a total of 23 antennas located at a high-altitude site in eastern California. CARMA operates at three bands: $1 \mathrm{~cm}(27-35 \mathrm{GHz}), 3 \mathrm{~mm}(85-116 \mathrm{GHz})$, and $1 \mathrm{~mm}$ (215-270 GHz), and consists of six $10.4 \mathrm{~m}$, nine $6.1 \mathrm{~m}$, and eight $3.5 \mathrm{~m}$ antennas that are combined to form the full CARMA array. The data presented in this analysis were observed in the $1 \mathrm{~cm}$ band with fifteen $500 \mathrm{MHz}$ channels between 27.5 and $35 \mathrm{GHz}$ using the eight $3.5 \mathrm{~m}$ antennas. Six of the eight antennas formed a compact configuration with baseline lengths of $\sim 0.3-2.0 \mathrm{k} \lambda$, while the remaining two antennas provided much longer baselines of $\sim 2.0-8.0$ $\mathrm{k} \lambda$. We refer to data from the baselines between the six compact antennas as short-baseline data, and data from the baselines between the six compact antennas and the two outlying antennas as longbaseline data. In Fig. 1, we plot the Fourier space $u$ - $v$ coverage for our observations of one of our targets, ECC181. The $u$-v coverage of the rest of our 15 targets is very similar. In the left-hand panel of Fig. 1 we plot the complete $u-v$ coverage for all baselines, which clearly illustrates how we separate the data into short- and longbaseline data, while in the right-hand panel we plot a zoomed-in view of the short baselines.

Our CARMA observations were performed during three separate observing runs in 2012 May, 2012 August, and 2013 January. The data were reduced using a suite of MATLAB routines, which convert the data to physical units, correct for instrumental phase and amplitude variations, flag data that do not meet the designed criteria at each stage of reduction, and perform the data calibration (see Muchovej et al. 2007, for further details on the reduction pipeline). The absolute calibration is derived from observations of Mars, and based on long-term monitoring of flux density calibrators, we estimate that the calibration is accurate to 5 percent. The reduction pipeline outputs the calibrated visibility data, which were then imported into AIPS, where the task IMAGR was used to perform the Fourier transform and deconvolution. This task uses a CLEAN-based algorithm to perform the deconvolution, and we implemented a Briggs robust parameter of 0 , providing a compromise between uniform and natural weighting. We imaged the short-baseline data and the long-baseline data separately, using a 120 and 20 arcsec restoring beam (FWHM), respectively. The final CARMA maps are displayed in Fig. A1, and the rms noise in both the short-baseline $\left(\sigma_{\text {Short }}\right)$ and long-baseline $\left(\sigma_{\text {Long }}\right)$ images for each source is tabulated in Table 1.

The long-baseline data are ideal for identifying compact radio sources $(\lesssim 1$ arcmin), while the short-baseline data are sensitive to the more diffuse emission on angular scales of $\sim 2-12$ arcmin. Looking at the maps in Fig. A1, we detected four compact sources that are visible in both the short- and long-baseline data (one source each in the ECC189, ECC191, ECC340, and ECC346 maps). Using the AIPS task JMFIT, we fitted two-dimensional Gaussians to each source to derive positions and flux densities. We searched the literature to identify these sources, and the NVSS counterpart for each source is listed in Table 2. Also listed in Table 2 are the measured CARMA $1 \mathrm{~cm}$ flux densities. The CARMA flux density uncertainties include a 5 per cent calibration uncertainty combined in quadrature with the uncertainty from the fit to the source. Throughout the rest of this analysis, we ignore these four point sources.

In addition to the point sources, there is not much extended emission present in the short-baseline maps displayed in Fig. A1. In fact, we find that only 1 of our 15 clumps, ECC229, has a significant detection of emission, which consists of a more compact component to the west and a more extended structure, likely consisting of two separate components, to the east.

\subsection{Herschel data}

In order to characterize the physical conditions of the observed CARMA clumps, we used Herschel PACS and SPIRE data. Eight of our targets are covered by the Guaranteed Time Key Programme 'Probing the origin of the stellar initial mass function: a widefield Herschel photometric survey of nearby star-forming cloud 
Table 2. Flux densities of the four point sources identified in the CARMA maps.

\begin{tabular}{lccccrr}
\hline & \multicolumn{3}{c}{ Short baselines } & \multicolumn{3}{c}{ Long baselines } \\
Source & $\begin{array}{c}\text { RA } \\
(\mathrm{J} 2000)\end{array}$ & $\begin{array}{c}\text { Dec. } \\
(\mathrm{J} 2000)\end{array}$ & \multicolumn{1}{c}{$\begin{array}{c}S_{1 \mathrm{~cm}} \\
(\mathrm{mJy})\end{array}$} & $\begin{array}{c}\text { RA } \\
(\mathrm{J} 2000)\end{array}$ & \multicolumn{1}{c}{$\begin{array}{c}\text { Dec. } \\
(\mathrm{J} 2000)\end{array}$} & $\begin{array}{c}S_{1 \mathrm{~cm}} \\
(\mathrm{mJy})\end{array}$ \\
\hline NVSS 205303+682200 & $20: 53: 04.00$ & $+68: 22: 06.4$ & $2.85 \pm 0.64$ & $20: 53: 04.44$ & $+68: 21: 58.0$ & $3.45 \pm 0.65$ \\
NVSS 210243+675819 & $21: 02: 43.91$ & $+67: 58: 16.7$ & $101.77 \pm 5.30$ & $21: 02: 43.70$ & $+67: 58: 17.1$ & $102.52 \pm 5.27$ \\
NVSS 043015+541429 & $04: 30: 15.40$ & $+54: 14: 31.6$ & $4.41 \pm 0.62$ & $04: 30: 14.84$ & $+54: 14: 28.1$ & $4.32 \pm 0.69$ \\
NVSS 044819+530830 & $04: 48: 18.88$ & $+53: 08: 22.9$ & $10.26 \pm 1.40$ & $04: 48: 19.47$ & $+53: 08: 30.4$ & $9.98 \pm 1.07$ \\
\hline
\end{tabular}

complexes' (KPGT_pandre_1; André et al. 2010), while the remaining seven are covered by the Open Time Key Programme 'Galactic cold cores: a Herschel survey of the source populations revealed by Planck' (KPOT_mjuvela_1; Juvela et al. 2010).

The Herschel data were downloaded from the Herschel Data Archive and reprocessed using the Herschel Interactive Processing Environment (HIPE) version 10.0.2747. The PACS data were processed using the standard pipeline for extended sources. Starting with the level 1 PACS data, this processing performed a correction for the global correlated signal drift and produced the time-ordered data, which were used to create a map using the MADMAP map-maker. Finally, a point source artefact correction was applied, resulting in maps at 70, 100, and $160 \mu \mathrm{m}$ with an angular resolution of $\sim 6,7$, and 11 arcsec, respectively. The absolute calibration uncertainty of the PACS data was conservatively estimated to be 10 per cent. The SPIRE data were processed from level 1 to level 2.5 using the SPIA reduction routines, with the DESTRIPER map-maker used to produce maps at 250,350, and $500 \mu \mathrm{m}$ with an angular resolution of $\sim 18$, 25 , and 36 arcsec, respectively. The absolute calibration uncertainty of the SPIRE data was conservatively estimated to be 7 per cent.

Although PACS has three channels (70, 100, and $160 \mu \mathrm{m})$, only two (either 70 or $100 \mu \mathrm{m}$ along with $160 \mu \mathrm{m}$ ) can be observed simultaneously for each observation. Therefore, to ensure that observations in all three PACS channels are obtained, a few of our sources had both PACS/SPIRE parallel observations and PACS photometry observations. However, this not only results in observations in all three PACS channels, it also results in a duplication of observations in the $160 \mu \mathrm{m}$ channel. Not all of our sources had duplicate $160 \mu \mathrm{m}$ observations, but for the ones that did, we used the mean of the two $160 \mu \mathrm{m}$ maps. All 15 sources did have at least one set of PACS and SPIRE observations, meaning that we have Herschel observations at $160,250,350$, and $500 \mu \mathrm{m}$ for all of our sources which, as we will discuss in Section 4.1, is adequate for our analysis.

\section{CLUMP PROPERTIES}

To understand why only 1 of our 15 clumps had detectable $1 \mathrm{~cm}$ emission, it is crucial to explore the physical conditions of our sample. Properties such as the density and radiation field are important parameters in spinning up the grains to produce spinning dust emission. Additionally, we need to determine the evolutionary stage of the clumps, specifically, whether these sources are gravitationally bound and, if so, whether they are pre-stellar or protostellar. If indeed some of the clumps are protostellar, we also need to identify the location of young stellar objects (YSOs) as they can generate emission at $\mathrm{cm}$ wavelengths due to a variety of mechanisms such as stellar winds and/or shock-induced ionization, which can mimic the spinning dust emission spectral signal. Additionally, knowledge about the evolutionary stage of the clumps and, in particular, understanding if the observed sources are both simultaneously forming stars and harbouring spinning dust emission can help to understand the potential role of spinning dust emission in the star formation process.

Therefore, in Section 4.1 we compute the hydrogen column density and dust temperature within our clumps, and in Section 4.2 we estimate the hydrogen number density and the radiation field. Then, in Section 4.3 we estimate the mass and determine if the clump is stable or likely to undergo collapse, and in Section 4.4 we identify and classify candidate YSOs in the vicinity of our clumps.

\subsection{Hydrogen column density and dust temperature}

All of the Herschel maps were convolved to the angular resolution of the $500 \mu \mathrm{m}$ map using the convolution kernels produced by Aniano et al. (2011) to allow for accurate comparisons between each of the Herschel bands. To ensure we are probing the dense cores themselves and are not contaminated by foreground or background emission, we performed a background subtraction on the Herschel maps. For each of the Herschel maps, we computed the median value of the flux in a reference position that is devoid of emission, and subtracted this from the map. The emission in these backgroundsubtracted Herschel maps was then modelled using

$I_{v}=\mu m_{\mathrm{H}} N_{\mathrm{H}} \kappa_{v} B_{v}\left(T_{\mathrm{d}}\right)$,

where $I_{v}$ is the intensity at frequency $v, \mu$ is the molecular weight per hydrogen atom, which we assume to be $1.4, m_{\mathrm{H}}$ is the mass of an $\mathrm{H}$ atom, $N_{\mathrm{H}}$ is the hydrogen column density, $\kappa_{v}$ is the dust opacity, and $B_{v}\left(T_{\mathrm{d}}\right)$ is the Planck function for dust temperature $T_{\mathrm{d}}$. The dust opacity is the subject of much debate, with large variations observed between models, depending on the physical properties of the dust grains such as size, composition, and structure (e.g. Ossenkopf \& Henning 1994). In this analysis, we use the dust opacity parametrization defined by Beckwith et al. (1990):

$\kappa_{v}=0.1\left(\frac{v}{1000 \mathrm{GHz}}\right)^{\beta} \mathrm{cm}^{2} \mathrm{~g}^{-1}$,

which is applicable to these cold, dense environments (e.g. WardThompson et al. 2010; Planck Collaboration XXII 2011; Planck Collaboration XXIII 2011; Juvela et al. 2012). This normalization assumes a standard gas to dust mass ratio of 100 .

We used the model defined by equations (1) and (2) to fit the background-subtracted Herschel maps at 160, 250, 350, and $500 \mu \mathrm{m}$. We excluded the 70 and $100 \mu \mathrm{m}$ Herschel maps from the fit because our clumps are cold $(<14 \mathrm{~K}$; this was one of the selection criteria used to produce the Planck ECC catalogue), and therefore they do not emit strongly at wavelengths $\leq 100 \mu \mathrm{m}$. Furthermore, excluding the 70 and $100 \mu \mathrm{m}$ maps ensures that we avoid possible contamination from stochastically heated small dust grains. We inspected the 70 and $100 \mu \mathrm{m}$ maps for all of the clumps and confirmed that there is very little emission present at these wavelengths, justifying our decision to exclude them from the fit. 
Table 3. Derived properties of the cores analysed in this work, including the core size, mean column density $\left(\bar{N}_{\mathrm{H}}\right)$, mean density $\left(\bar{n}_{\mathrm{H}}\right)$, mean dust temperature $\left(\bar{T}_{\mathrm{d}}\right)$, mean radiation field $\left(\bar{G}_{0}\right)$, mass $(M)$, Bonnor-Ebert mass $\left(M_{\mathrm{BE}}\right)$, associated YSOs (YSO), observed $1 \mathrm{~cm}$ flux density $\left(S_{1 \mathrm{~cm}}^{\text {observed }}\right)$, and the predicted $1 \mathrm{~cm}$ spinning dust emission $\left(S_{1 \mathrm{~cm}}^{\text {predicted }}\right)$.

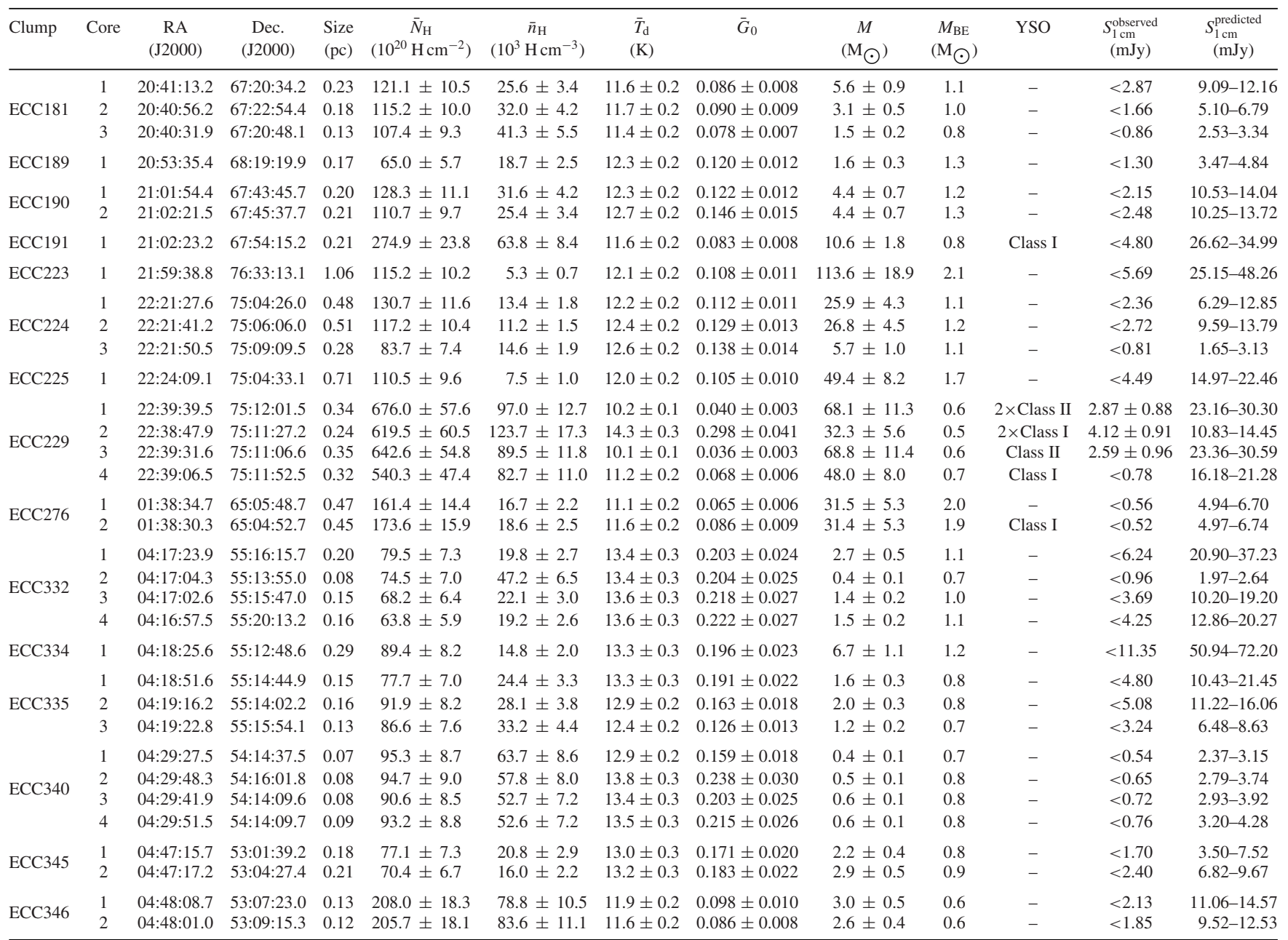

We fitted the data for $N_{\mathrm{H}}$ and $T_{\mathrm{d}}$, fixing the dust opacity index $\beta=2$ (e.g. Planck Collaboration XXII 2011; Planck Collaboration XXIII 2011; Juvela et al. 2012). This fit was performed on a pixelby-pixel basis using MPFIT (Markwardt 2009) resulting in maps of $N_{\mathrm{H}}$ and $T_{\mathrm{d}}$, along with their associated uncertainty maps, which were computed from the covariance matrix. The $N_{\mathrm{H}}$ and $T_{\mathrm{d}}$ maps for all 15 clumps are displayed in Fig. B1. For our 15 targets, we find values of $N_{\mathrm{H}}$ ranging from $\sim 6 \times 10^{21}$ to $\sim 7 \times 10^{22} \mathrm{H} \mathrm{cm}^{-2}$ and dust temperatures between $T_{\mathrm{d}} \sim 10$ and $\sim 15 \mathrm{~K}$. These values are well within the range found for the entire C3PO catalogue by Planck Collaboration XXIII (2011), and are consistent with those estimated by Juvela et al. (2012) using Herschel SPIRE data for their sample of cores.

\subsection{Hydrogen number density and radiation field}

Herschel, with its sub-arcmin angular resolution, is able to resolve the sources observed by Planck into sub-structures. In the case of the ECC clumps, we can therefore identify individual cores within each clump. For this purpose, we used the CLUMPFInd package (Williams, de Geus \& Blitz 1994), which identifies local peaks in the $N_{\mathrm{H}}$ maps and follows them down to lower levels, resulting in a decomposition of the map into multiple cores. CLUMPFIND identified 34 cores, the positions of which are listed in Table 3 and labelled in Fig. B1. In addition to the position, CLUMPFIND also computes the angular size of each identified core. Since this angular size is the effective circular radius, $R\left(=\left(N_{\text {pixels }} / \pi\right)^{1 / 2}\right.$, where $N_{\text {pixels }}$ is the total number of pixels), we assumed that the cores are spherical, and for each core we computed the mean column density, $\bar{N}_{\mathrm{H}}$, and the mean dust temperature, $\bar{T}_{\mathrm{d}}$. The values of $\bar{N}_{\mathrm{H}}$ and $\bar{T}_{\mathrm{d}}$ for each core are listed in Table 3.

Using the distance to each clump (see Table 1), we converted the angular size of each core computed by CLUMPFIND to a physical, linear size, $L$. Since we have performed a background/foreground subtraction, by combining $\bar{N}_{\mathrm{H}}$ and the linear size we can estimate the mean density, $\bar{n}_{\mathrm{H}}$. Based on the conservation of mass, the total mass in the $N_{\mathrm{H}}$ map within a given radius, $R$, is equivalent to the mass within a sphere of the same radius, with a mean density, $\bar{n}_{\mathrm{H}}$, i.e.

$\pi R^{2} \mu m_{\mathrm{H}} \bar{N}_{\mathrm{H}}=\frac{4}{3} \pi R^{3} \mu m_{\mathrm{H}} \bar{n}_{\mathrm{H}}$.

Since $R=L / 2$, this results in

$\bar{n}_{\mathrm{H}}=\frac{3}{2} \frac{\bar{N}_{\mathrm{H}}}{L}$. 
Using equation (4), we estimated $\bar{n}_{\mathrm{H}}$ for each core. The uncertainty on $\bar{n}_{\mathrm{H}}$ was calculated assuming a 10 percent uncertainty on the distance. The derived $\bar{n}_{\mathrm{H}}$ values range from $\sim 5 \times 10^{3} \mathrm{H} \mathrm{cm}^{-3}$ in core 1 in ECC223 to $\sim 125 \times 10^{3} \mathrm{H} \mathrm{cm}^{-3}$ in core 2 in ECC229 (see Table 3). Although these values are mean quantities, they are comparable to the densities estimated in the entire C3PO catalogue (Planck Collaboration XXIII 2011).

In addition to estimating $\bar{n}_{\mathrm{H}}$, we also estimated the mean radiation field, $\bar{G}_{0}$, in each core by converting $\bar{T}_{\mathrm{d}}$ into $\bar{G}_{0}$ using

$\bar{G}_{0}=\left(\frac{\bar{T}_{\mathrm{d}}}{17.5 \mathrm{~K}}\right)^{4+\beta}$,

where we fixed $\beta=2$ as before. The computed values of $\bar{G}_{0}$ are also listed in Table 3 and we find values ranging from $\sim 0.04$ to 0.30 , where a value of 1 corresponds to the Mathis, Mezger \& Panagia (1983) solar neighbourhood radiation field. Such low values of $\bar{G}_{0}$ indicate that the interior of these dense environments are shielded from the surrounding interstellar radiation field.

\subsection{Mass}

We estimated the total (dust+gas) mass for each of our cores using equation (3). The computed mass estimates are listed in Table 3 and range between $\sim 0.4$ and $115 \mathrm{M}_{\odot}$.

To determine if our cores are gravitationally stable or in the process of collapsing, we also computed the Bonnor-Ebert mass, $M_{\mathrm{BE}}$, the mass of an isothermal sphere in hydrostatic and pressure equilibrium. Cores with $M>M_{\mathrm{BE}}$ are unstable, and will therefore collapse, while cores with $M<M_{\mathrm{BE}}$ are stable. We estimated the Bonnor-Ebert mass as

$M_{\mathrm{BE}} \approx 1.82\left(\frac{\bar{n}_{\mathrm{H}}}{10^{4} \mathrm{H} \mathrm{cm}^{-3}}\right)^{-0.5}\left(\frac{T_{\text {gas }}}{10 \mathrm{~K}}\right)^{1.5} \mathrm{M}_{\odot}$

from Lada et al. (2008). Both the values of $M$ and $M_{\mathrm{BE}}$ for all of the cores are listed in Table 3 . We find that 29 of our 34 cores are unstable, and therefore undergoing collapse.

\subsection{Young stellar objects}

Since the unstable cores will collapse and eventually form stars, we also investigated the ongoing star formation by searching for YSOs in the vicinity of our cores. Various IR colour-cuts have been used to identify YSOs, e.g. based on Spitzer (e.g. Allen et al. 2004) and WISE (e.g. Koenig et al. 2012) data. Since not all of our clumps have been observed with Spitzer, we take advantage of the all-sky coverage of WISE, which mapped the entire sky simultaneously at four wavelengths: 3.4, 4.6, 12, and $22 \mu \mathrm{m}$ (Wright et al. 2010). We apply the colour-colour selection criteria developed by Koenig et al. (2012) to the AllWISE all-sky source catalogue to identify YSO candidates, excluding sources with a signal-to-noise ratio $(\mathrm{SNR})<5$ and contaminated sources identified by the catalogue contamination flags ' $\mathrm{D}$ ' (diffraction spikes), 'P' (persistent latent artefacts), 'H' (haloes from bright sources), and 'O' (optical ghosts from bright sources). This selection criteria allows us to reject extragalactic contaminants and distinguish between class I and class II YSO candidates (for details of the specific selection criteria, see appendices A.1, A.2, and A.3 in Koenig et al. 2012).

This analysis enabled us to identify YSO candidates associated with each clump. We found that only 5 of our 15 clumps have at least one YSO candidate within our $20 \operatorname{arcmin} \times 20 \operatorname{arcmin}$ maps displayed in Fig. B1, and that only four of these (ECC190, ECC191, ECC229, and ECC276) have a YSO candidate within the CARMA primary beam. The location of the YSO candidates, and their classification (either class I or class II), are marked on Fig. B1. It is widely accepted that no colour-colour identification criteria are 100 percent accurate. Using the contamination rate of false identifications from Koenig et al. (2012), we estimate that there are $\sim 0.4-0.9$ false identifications in each of our maps.

Looking at the location of these YSO candidates relative to our cores, we determined that six cores are possibly associated with a YSO (see Table 3). Core 1 in ECC191 is coincident with a class I YSO candidate, while cores 2, 3, and 4 in ECC229 are coincident with two class I YSO candidates, a class II YSO candidate, and a class I YSO candidate, respectively. In addition, there are two cores which have YSO candidates nearby: core 1 in ECC229 has two nearby class II YSO candidates, and core 2 in ECC276 has a class I YSO candidate nearby.

YSOs have previously been detected at $\mathrm{cm}$ wavelengths (e.g. AMI Consortium: Scaife et al. 2011), but of the six cores that are possibly associated with a YSO candidate, only three have detectable $\mathrm{cm}$ emission (ECC229 cores 1, 2, and 3). However, given the variety of mechanisms by which YSOs can produce $\mathrm{cm}$ emission, perhaps it is not surprising that we do not detect $\mathrm{cm}$ emission from all of our candidates. This also makes it more difficult to interpret the $\mathrm{cm}$ emission that we detect in ECC229, as we will discuss in Section 5.1 .

\section{DISCUSSION}

\subsection{ECC229}

Out of all of our 15 clumps, ECC229 is the only clump for which we detect any extended $\mathrm{cm}$ emission, making it particularly interesting. In our CARMA map of ECC229, we detected three components of $\mathrm{cm}$ emission (associated with cores 1, 2, and 3), although we identified four cores. Based on the values listed in Table 3, the cores in ECC229 have the highest values of $\bar{N}_{\mathrm{H}}$. Moreover, as shown in Fig. B1, the cm emission appears to be spatially correlated with $N_{\mathrm{H}}$ and originating from the densest regions.

Core 2 in ECC229 has the highest density $\left(\bar{n}_{\mathrm{H}}=(123.7 \pm\right.$ 17.3) $\times 10^{3} \mathrm{H} \mathrm{cm}^{-3}$ ), but also the warmest dust temperature $\left(\bar{T}_{\mathrm{d}}=14.3 \pm 0.3 \mathrm{~K}\right)$ and highest radiation field value $\left(\bar{G}_{0}=0.298 \pm 0.041\right)$, potentially suggesting that the star formation process has already begun. Indeed two YSO candidates, likely heating the dust in the core, are found to be associated with core 2 . In fact all four of the cores in ECC229 appear to be associated with a YSO candidate, and the fact that we only detect $\mathrm{cm}$ emission from three of the cores might suggest that the $\mathrm{cm}$ emission is not linked to the presence of the YSOs. However, given that YSOs can produce $\mathrm{cm}$ emission via a variety of different mechanisms (accretion, stellar winds, shock ionization), it is far from clear. For example, the spectral index at cm wavelengths (defined as $\alpha$, where $S_{\nu} \propto \nu^{\alpha}$ ) predicted from free-free emission from an ionized stellar wind can cover the range from $-0.1 \leq \alpha \leq+2.0$, with typical values of $\alpha$ $\sim 0.6$ in the case of a spherical, isothermal stellar wind (Reynolds 1986). A similar range of values for $\alpha$ is produced for free-free emission from accretion and shock ionization. The spectral index from spinning dust emission at these wavelengths is dependent on the frequency of the peak of the emission, and so $\alpha$ can be either positive or negative. This makes it difficult to distinguish between the $\mathrm{cm}$ emission arising from YSOs as opposed to spinning dust emission, especially in the absence of multiwavelength $\mathrm{cm}$ observations, which can closely trace the microwave emission, combined with IR 

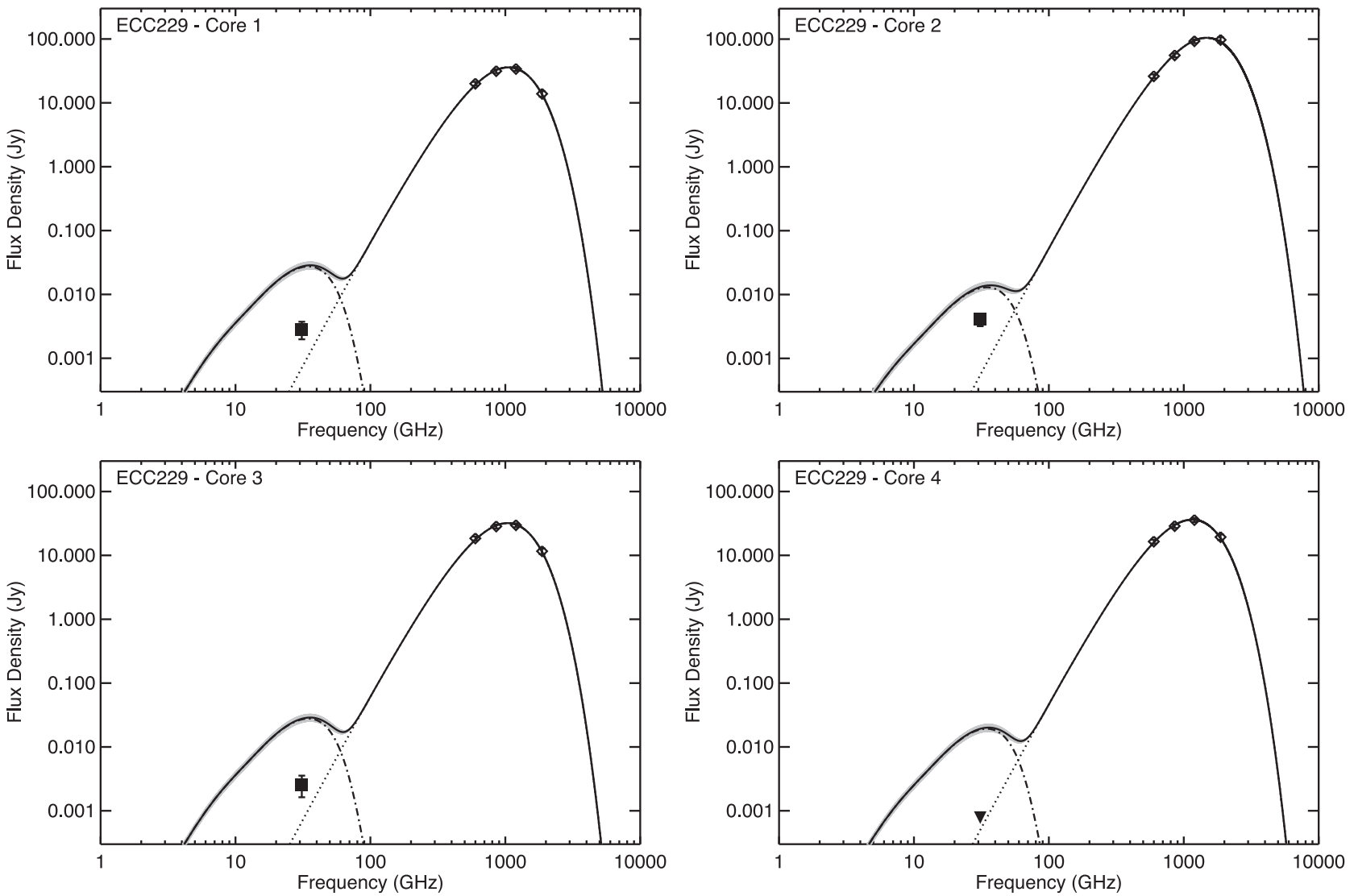

Figure 2. Spectrum for the four cores in ECC229, consisting of both a spinning dust (dot-dashed line) and thermal dust (dotted line) component. The thermal dust emission is modelled using a modified blackbody with $\beta$ fixed at 2 , and is fitted to the integrated flux density at $160,250,350$, and $500 \mu \mathrm{m}$ (diamonds) in each core, while the spinning dust curve, and associated uncertainty (shaded region), is the predicted level of spinning dust emission based on the modelling analysis. Also plotted is the measured flux density at $1 \mathrm{~cm}$ (for core 4 in which we do not detect any cm emission, we have plotted the $5 \sigma$ upper limit), illustrating how the predicted spinning dust emission is larger than what is observed with CARMA.

spectroscopic data, which can provide details on the YSO geometry (e.g. disc inclination, etc.). Therefore, lacking this ancillary information, to determine the presence of spinning dust emission (or a lack thereof), we compared our CARMA observations to the predicted level of spinning dust emission based on the physical conditions in each core.

\subsection{Spinning dust emission}

With the physical properties of the clumps characterized, as described in Section 4, we can investigate the significance of our CARMA observations, namely: does the fact that we did not detect $\mathrm{cm}$ emission in 14 of our 15 clumps signify that there is no spinning dust emission present? In order to address this question, we computed the expected level of spinning dust emission, using the constraints provided by the analysis of the Herschel data.

We characterized our cores by using the $\bar{n}_{\mathrm{H}}$ and $\bar{G}_{0}$ values derived in Section 4.2, the gas temperatures, $T_{\text {gas }}$, listed in Table 1, and the standard values for dense environments for the hydrogen ionization fraction $\left(x_{\mathrm{H}}=0\right)$, the carbon ionization fraction $\left(x_{\mathrm{C}}=10^{-6}\right)$, and the molecular hydrogen fraction $(y=0.999)$ as defined by Draine $\&$ Lazarian (1998). In addition to these parameters, and since these are dense environments, we used the Weingartner \& Draine (2001) grain size distribution for $R_{\mathrm{V}}=5.5$ with $b_{\mathrm{C}}=3 \times 10^{-5}$ (corresponding to the parameters in Weingartner \& Draine 2001, table 1, line 16). With these parameters as inputs, we used the spinning dust model, SPDUST (Silsbee, Ali-Haïmoud \& Hirata 2011) to predict the expected level of spinning dust emission at $1 \mathrm{~cm}$.

SPDUST estimates the spinning dust emissivity in units of $\mathrm{Jy} \mathrm{sr}^{-1}$ $\mathrm{H}^{-1} \mathrm{~cm}^{2}$ which we converted to a flux by using our computed value of $\bar{N}_{\mathrm{H}}$. To estimate the uncertainty on the predicted spinning dust emission, we repeated this modelling analysis 1000 times, randomising the input $\bar{n}_{\mathrm{H}}$ and $\bar{G}_{0}$ values within their uncertainty, resulting in a range of predicted values for the spinning dust emission at $1 \mathrm{~cm}$. We then compared the predicted spinning dust emission with our CARMA maps. For the cores for which we do not detect any $\mathrm{cm}$ emission we computed a conservative $5 \sigma$ upper limit by scaling the rms noise in the CARMA maps based on the ratio of the size of the beam to the size of the core, while for ECC229, we fitted the extended emission with three two-dimensional Gaussian components using the AIPS task JMFIT. The last two columns in Table 3 list our measured $1 \mathrm{~cm}$ flux densities $\left(S_{1 \mathrm{~cm}}^{\text {observed }}\right)$ along with the predicted levels of the spinning dust emission $\left(S_{1 \mathrm{~cm}}^{\text {predicted }}\right)$. For all of our cores we find that the predicted level of spinning dust emission is much larger than what we observe in our CARMA maps. We 
illustrate this result for the four cores in ECC229 in Fig. 2 , where we plot the predicted spinning dust emission, with its associated uncertainty, the thermal dust emission, modelled using a modified blackbody with $\beta$ fixed at 2, and our CARMA $1 \mathrm{~cm}$ flux density measurements.

We do note, however, that this result depends strongly on the spinning dust modelling analysis. First, since we are only comparing our observed $\mathrm{cm}$ emission with the predicted spinning dust emission, we are actually being conservative as we are ignoring any possible contribution to the $1 \mathrm{~cm}$ emission from thermal dust emission or free-free emission, which would increase the expected level of the $1 \mathrm{~cm}$ emission. Secondly, it is possible that the discrepancy between the observed and the predicted level of spinning dust emission could be due to an incorrect assumption in our spinning dust modelling. For example, there is much uncertainty on the value of the electric dipole moment of dust grains. The value used in this analysis is consistent with the standard value estimated by Draine \& Lazarian (1998) - an average rms dipole moment per atom of $0.38 \mathrm{D}$. If this value is overestimated, it would result in an overestimate of the level of the spinning dust emission. Likewise, we might have overestimated $\bar{n}_{\mathrm{H}}$, as a consequence of the uncertainty in the distance estimate or due to our assumption that the core geometry is spherical. Similarly, our estimate of $\bar{T}_{\mathrm{d}}$, and hence $\bar{G}_{0}$, may be overestimated due to the mixing of different line-of-sight temperature variations (e.g. Nielbock et al. 2012; Roy et al. 2014). Again, such overestimates would result in us overestimating the spinning dust emission. Finally, our adopted grain size distribution might not be appropriate for Galactic cores. Although we used the grain size distribution for dense environments with $R_{\mathrm{V}}=5.5$ (as opposed to values of $R_{\mathrm{V}}=3.1$ or $R_{\mathrm{V}}=4.0$ ), this could still be overestimating the abundance of the small grains responsible for the spinning dust emission. In a forthcoming paper (Tibbs et al. 2015), we will investigate these scenarios in more detail and, in particular, use these observations to place a constraint on the abundance of the small dust grains in our sample of cores.

Finally, we stress that the sample of Galactic cores which was the subject of this study does not span the full parameter space (in terms of density, temperature, mass, etc.) relative to the Galactic cold core population. For this reason, the conclusions reached by our analysis cannot easily be generalized, and more $\mathrm{cm}$ observations like the ones presented are needed to allow confirmation of our findings.

\section{CONCLUSIONS}

We have attempted, for the first time, to search for spinning dust emission in a sample of Galactic cold clumps. To do this we observed a sample of 15 cold clumps with CARMA at $1 \mathrm{~cm}$, and found that only 1 of our 15 clumps exhibited significant extended emission. To determine if the lack of detection of $1 \mathrm{~cm}$ emission could rule out a spinning dust detection, we investigated the physical properties of our sample of clumps using ancillary Herschel and WISE data. Using Herschel photometric data at 160, 250, 350, and $500 \mu \mathrm{m}$ of our 15 cold clumps we produced maps of $N_{\mathrm{H}}$ and $T_{\mathrm{d}}$, from which we identified 34 cores, characterized by the densest and coldest regions within each of the 15 clumps. For each core we estimated $\bar{N}_{\mathrm{H}}, \bar{T}_{\mathrm{d}}, \bar{n}_{\mathrm{H}}$, and $\bar{G}_{0}$. Making use of the all-sky coverage of WISE, we used the AllWISE source catalogue to identify candidate YSOs and found that 6 of our 34 cores were associated with a YSO candidate.

With the physical environments of each core constrained, we used SPDUST to model the spinning dust emission, which we compared to our CARMA observations, and we found that the observed $\mathrm{cm}$ emission in all of our cores was below the predicted level. This implies that we do not detect spinning dust emission from 14 of our 15 clumps, and in the one clump in which we do detect $\mathrm{cm}$ emission, it could be due to either spinning dust emission, but at a level much lower than predicted based on the modelling, or it could be due to free-free emission from YSOs.

This analysis is the first attempt to detect spinning dust emission in Galactic cores; however, we emphasize that our sample is not statistically representative of the entire Galactic cold core population, and therefore we are cautious to extend this result to all of the cold cores in the Galaxy.

\section{ACKNOWLEDGEMENTS}

We thank the anonymous referee for providing detailed comments that have improved the content of this paper.

This work has been performed within the framework of a NASA/ADP ROSES-2009 grant no. 09-ADP09-0059.

Support for CARMA construction was derived from the Gordon and Betty Moore Foundation, the Kenneth T. and Eileen L. Norris Foundation, the James S. McDonnell Foundation, the Associates of the California Institute of Technology, the University of Chicago, the states of California, Illinois, and Maryland, and the National Science Foundation. Ongoing CARMA development and operations are supported by the National Science Foundation under a cooperative agreement, and by the CARMA partner universities.

This research has made use of the NASA/IPAC Infrared Science Archive, which is operated by the Jet Propulsion Laboratory, California Institute of Technology, under contract with the National Aeronautics and Space Administration.

This publication makes use of data products from the Wide-field Infrared Survey Explorer, which is a joint project of the University of California, Los Angeles and the Jet Propulsion Laboratory/California Institute of Technology, and NEOWISE, which is a project of the Jet Propulsion Laboratory/California Institute of Technology. WISE and NEOWISE are funded by the National Aeronautics and Space Administration.

\section{REFERENCES}

Allen L. E. et al., 2004, ApJS, 154, 363

AMI Consortium: Scaife A. M. M. et al., 2011, MNRAS, 410, 2662

André P. et al., 2010, A\&A, 518, L102

Aniano G., Draine B. T., Gordon K. D., Sandstrom K., 2011, PASP, 123, 1218

Beckwith S. V. W., Sargent A. I., Chini R. S., Guesten R., 1990, AJ, 99, 924

Bergin E. A., Tafalla M., 2007, ARA\&A, 45, 339

Casassus S., Cabrera G. F., Förster F., Pearson T. J., Readhead A. C. S., Dickinson C., 2006, ApJ, 639, 951

Clemens D. P., 1985, ApJ, 295, 422

Draine B. T., Lazarian A., 1998, ApJ, 508, 157

Finkbeiner D. P., 2004, ApJ, 614, 186

Génova-Santos R. et al., 2015, MNRAS, 452, 4169

Harper S. E., Dickinson C., Cleary K., 2015, preprint (arXiv:1501.01484)

Hilton J., Lahulla J. F., 1995, A\&AS, 113, 325

Juvela M. et al., 2010, A\&A, 518, L93

Juvela M. et al., 2012, A\&A, 541, A12

Koenig X. P., Leisawitz D. T., Benford D. J., Rebull L. M., Padgett D. L., Assef R. J., 2012, ApJ, 744, 130

Lada C. J., Muench A. A., Rathborne J., Alves J. F., Lombardi M., 2008, ApJ, 672, 410

Leitch E. M., Readhead A. C. S., Pearson T. J., Myers S. T., 1997, ApJ, 486, L23 
Markwardt C. B., 2009, in Bohlender D. A., Durand D., Dowler P., eds, ASP Conf. Ser. Vol. 411, Astronomical Data Analysis Software and Systems XVIII. Astron. Soc. Pac., San Francisco, p. 251

Mathis J. S., Mezger P. G., Panagia N., 1983, A\&A, 128, 212

Muchovej S. et al., 2007, ApJ, 663, 708

Nielbock M. et al., 2012, A\&A, 547, A11

Ossenkopf V., Henning T., 1994, A\&A, 291, 943

Pilbratt G. L. et al., 2010, A\&A, 518, LL1

Planck Collaboration VII, 2011, A\&A, 536, A7

Planck Collaboration XX, 2011, A\&A, 536, A20

Planck Collaboration XXII, 2011, A\&A, 536, A22

Planck Collaboration XXIII, 2011, A\&A, 536, A23

Reynolds S. P., 1986, ApJ, 304, 713

Roy A. et al., 2014, A\&A, 562, A138
Silsbee K., Ali-Haïmoud Y., Hirata C. M., 2011, MNRAS, 411, 2750

Tibbs C. T. et al., 2010, MNRAS, 402, 1969

Tibbs C. T., Scaife A. M. M., Dickinson C., Paladini R., Davies R. D., Davis

R. J., Grainge K. J. B., Watson R. A., 2013, ApJ, 768, 98

Tibbs C. T. et al., 2015, MNRAS, submitted

Ward-Thompson D. et al., 2010, A\&A, 518, L92

Watson R. A., Rebolo R., Rubiño-Martín J. A., Hildebrandt S., Gutiérrez C. M., Fernández-Cerezo S., Hoyland R. J., Battistelli E. S., 2005, ApJ, 624, L89

Weingartner J. C., Draine B. T., 2001, ApJ, 548, 296

Williams J. P., de Geus E. J., Blitz L., 1994, ApJ, 428, 693

Wright E. L. et al., 2010, AJ, 140, 1868

Wu Y., Liu T., Meng F., Li D., Qin S.-L., Ju B.-G., 2012, ApJ, 756, 76

Ysard N., Juvela M., Verstraete L., 2011, A\&A, 535, A89 


\section{APPENDIX A: CARMA MAPS}
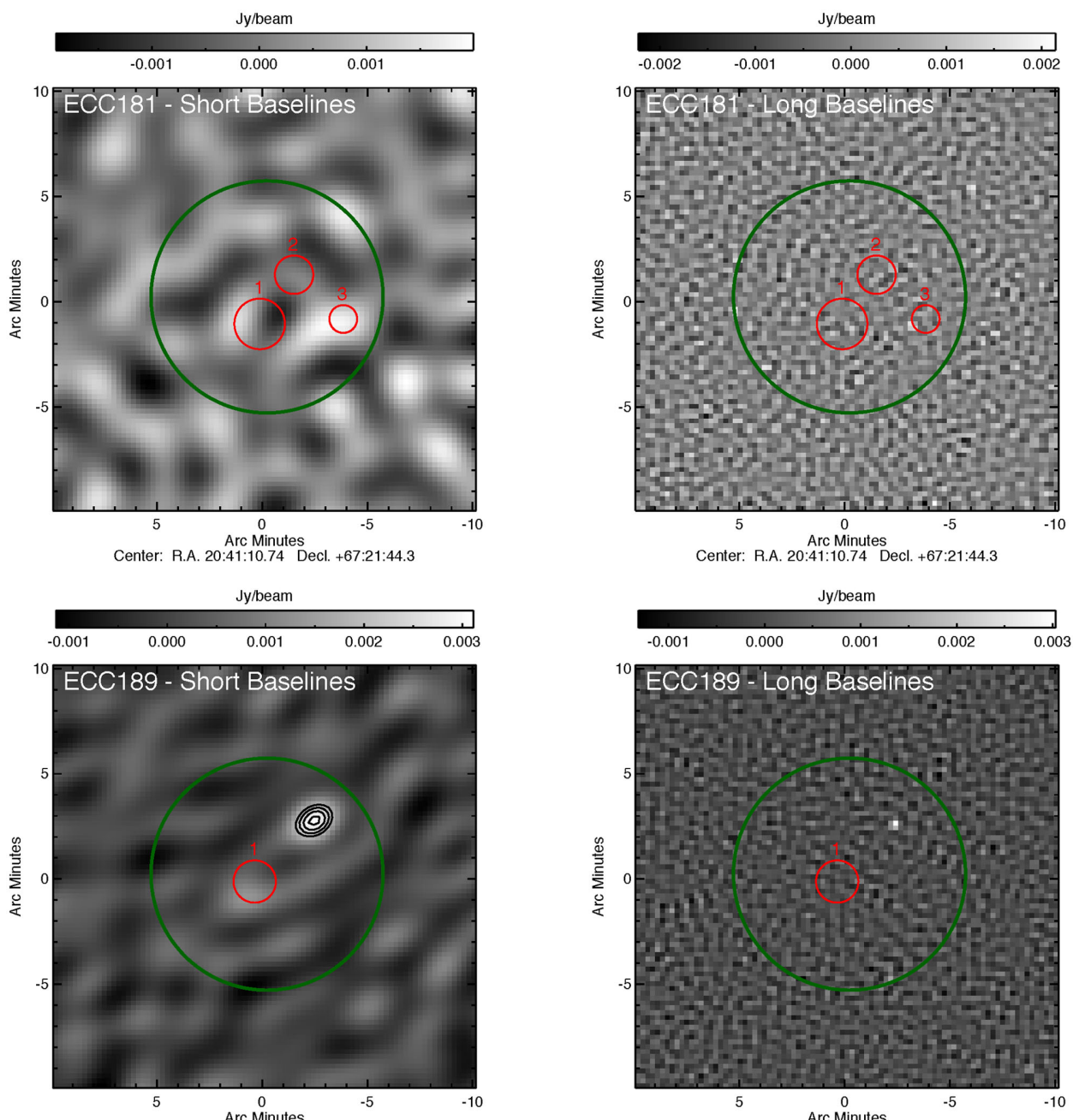

Center: R.A. 20:53:30.29 Decl. $+68: 19: 32.9$
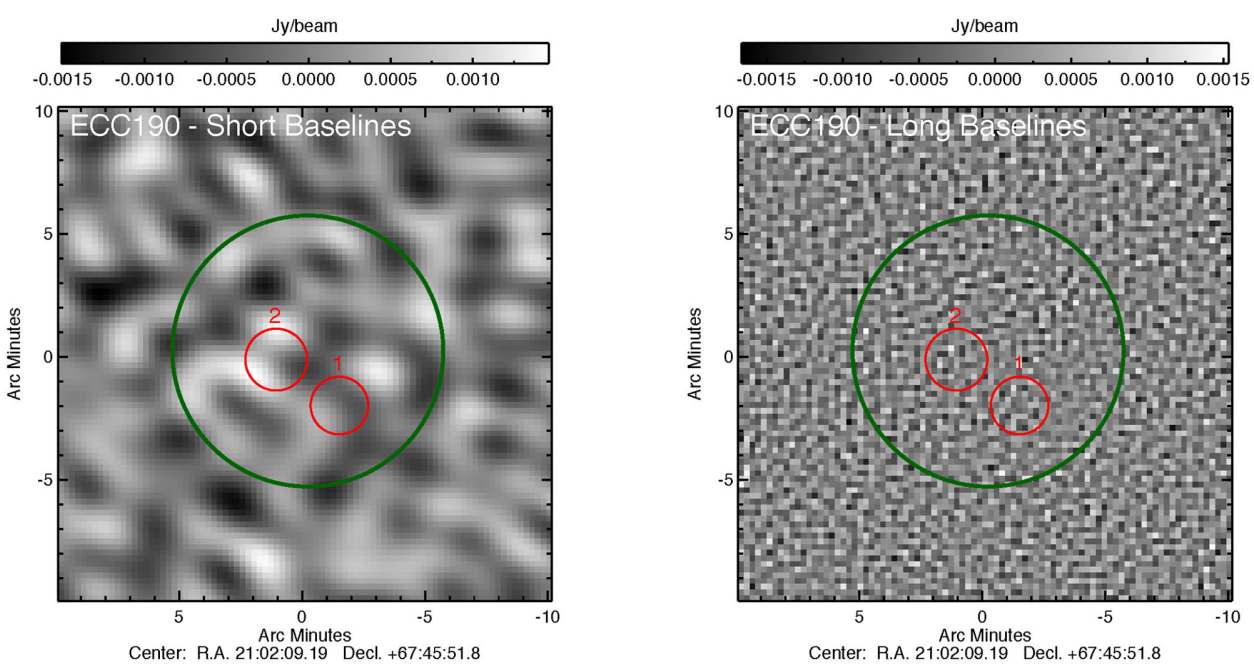

Figure A1. CARMA $1 \mathrm{~cm}$ maps for the short- (left) and long- (right) baseline data for all 15 cold clumps. The location of the cores are numbered and identified by red circles. CARMA contours, starting at $5 \sigma$ and increasing linearly, are displayed for the short-baseline data (except for ECC191 where the contours increase in steps of $10 \sigma$ ) along with the CARMA 11 arcmin primary beam (green circle). 


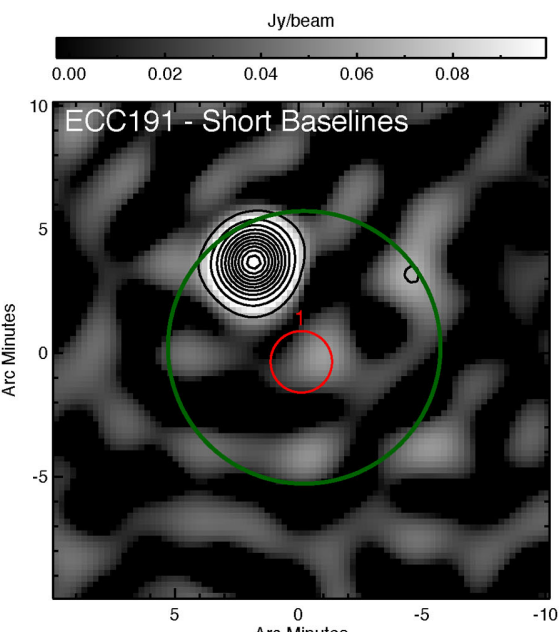

Arc Minutes
Center: R.A. 21:02:23.24 Decl. +67:54:43.3

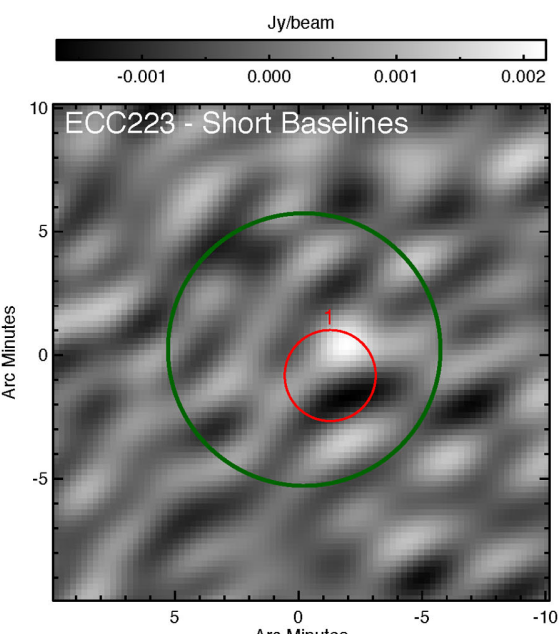

Arc Minutes
Center: R.A. 21:59:59.03 Decl. +76:34:08.7
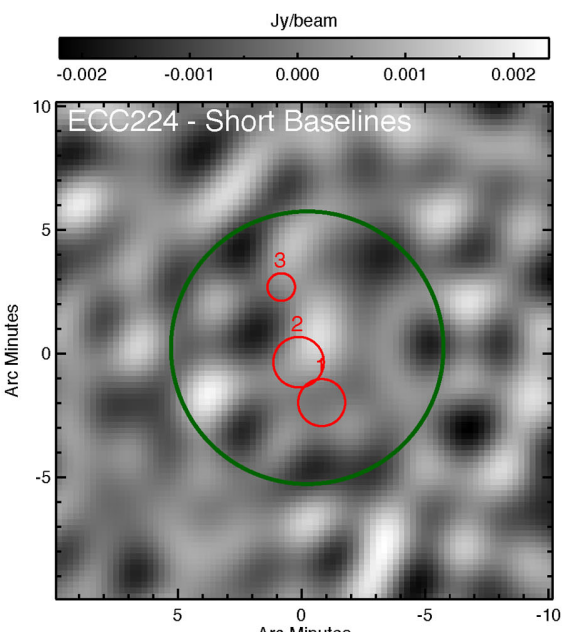

Arc Minutes
Center: R.A. 22:21:37.34 Decl. +75:06:33.5

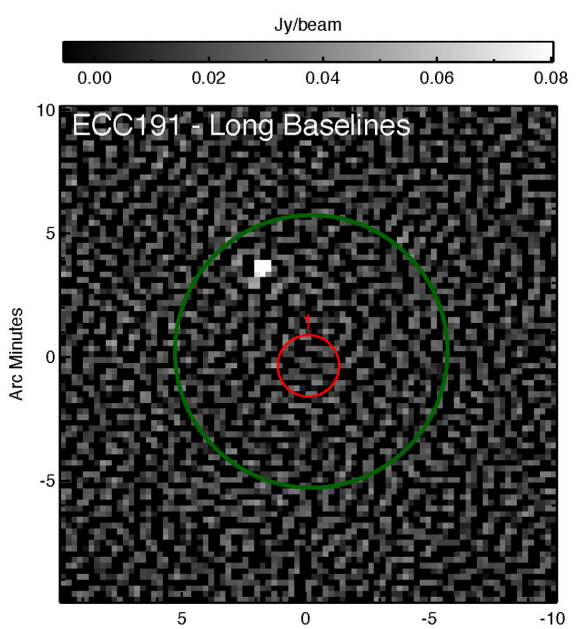

Arc Minutes
Center: R.A. 21:02:23.24 Decl. +67:54:43.3
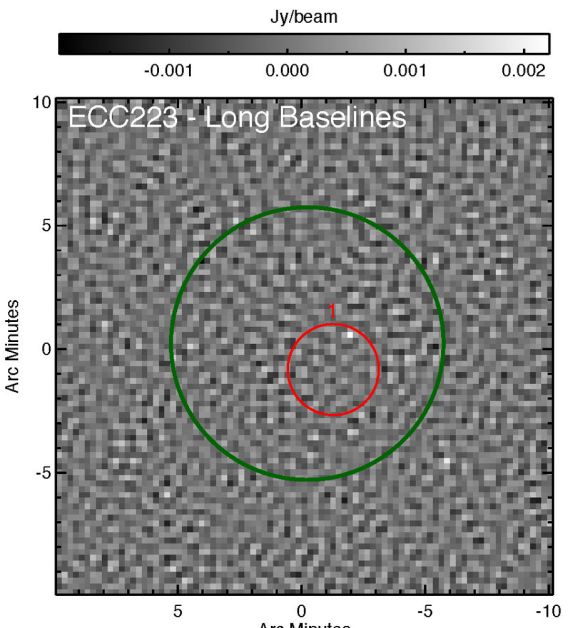

Arc Minutes
Center: R.A. 21:59:59.03 Decl. +76:34:08.7

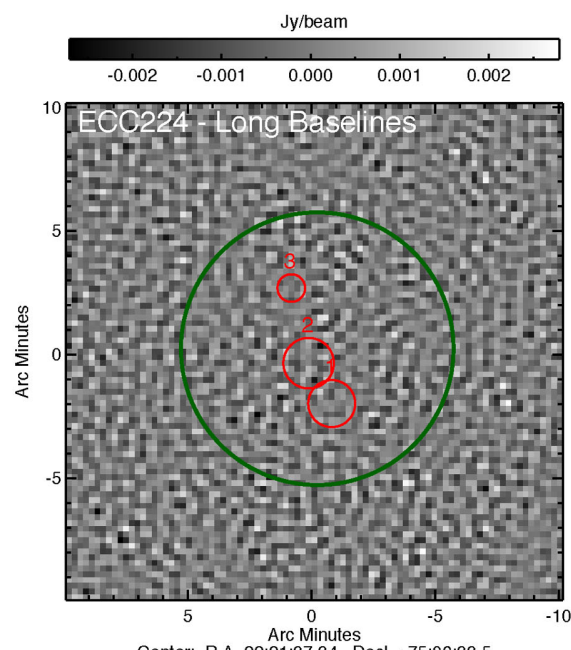

Arc Minutes
Center: R.A. 22:21:37.34 Decl. +75:06:33.5

Figure A1 - continued 

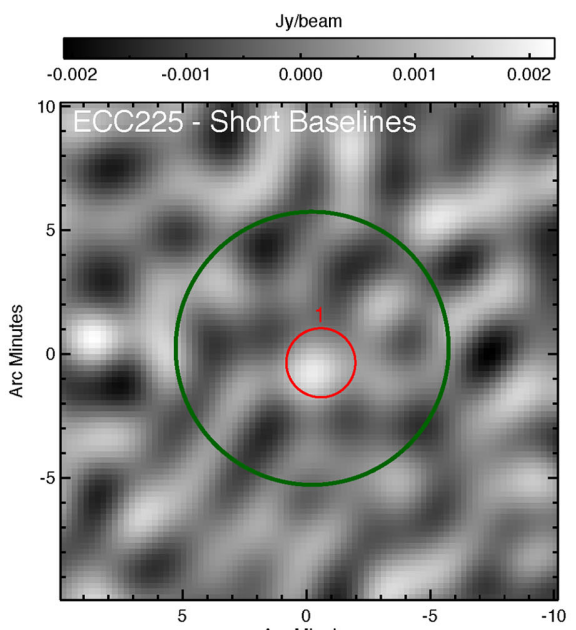

Arc Minutes
Center:

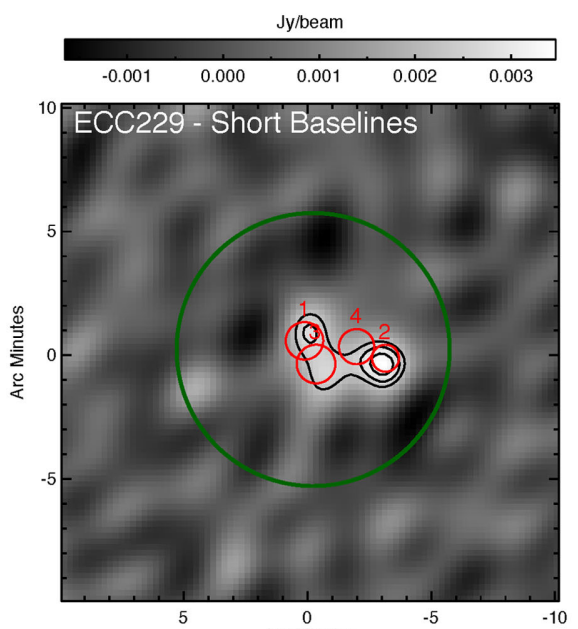

Arc Minutes
Center:
R.A. 22:39:35.57 Decl. $+75: 11: 34.0$
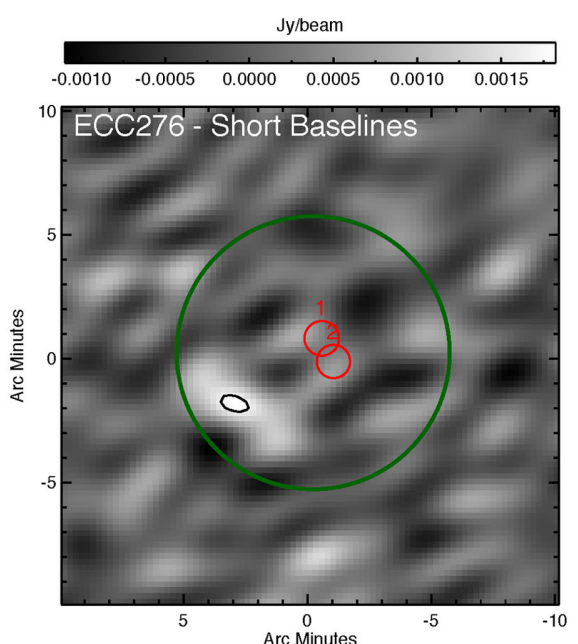

Arc Minutes
Center: R.A. 01:38:39.14 Decl. +65:05:06.5

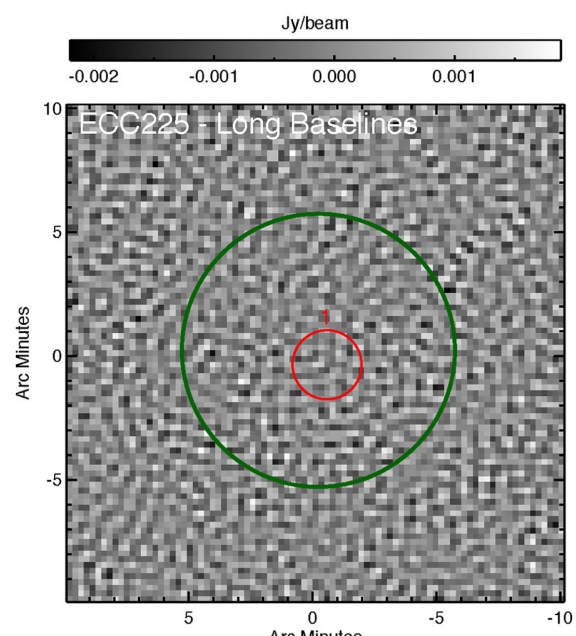

Arc Minutes
Center: R.A. $22: 24: 16.23$ Decl. $+75: 05: 01.8$
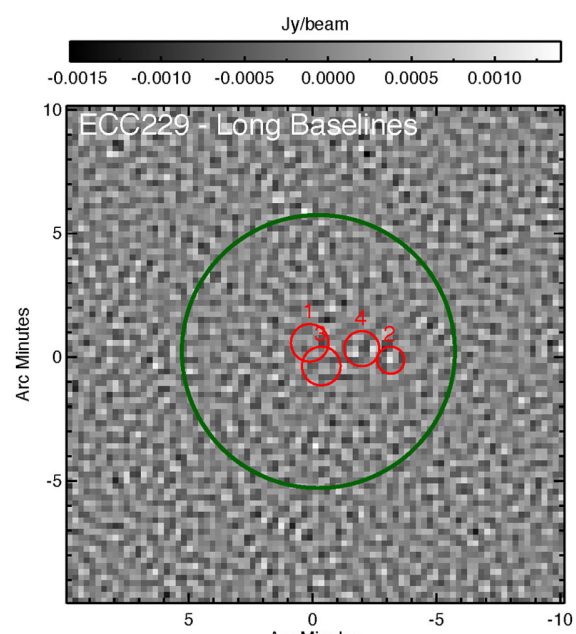

Arc Minutes
Center: R.A. 22:39:35.57 Decl. $+75: 11: 34.0$
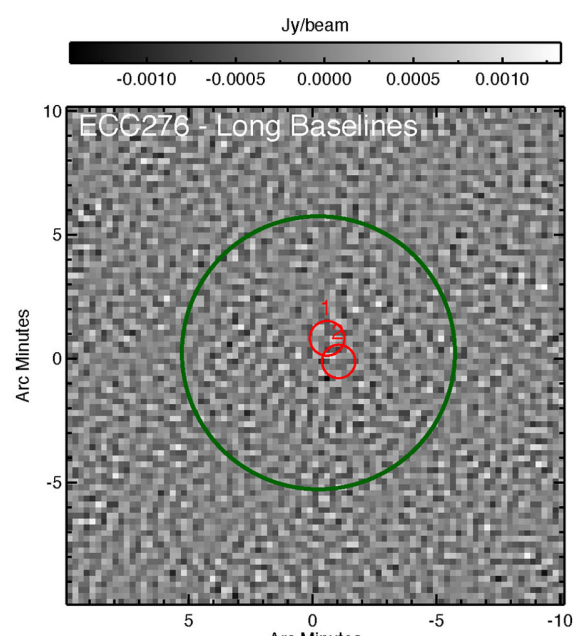

Arc Minutes
Center: R.A. 01:38:39.14 Decl. +65:05:06.5

Figure A1 - continued 


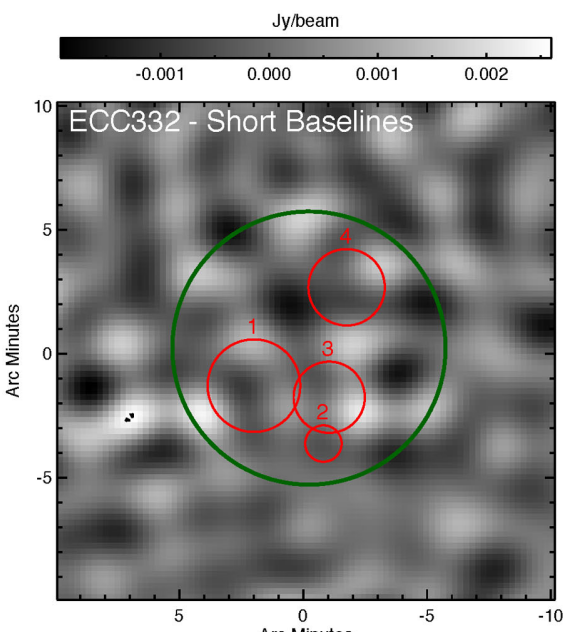

Arc Minutes
Center: R.A. 04:17:09.10 Decl. +55:17:39.4

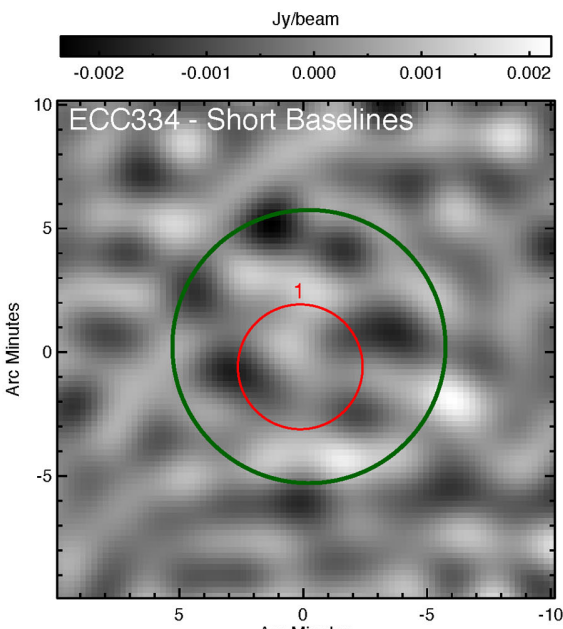

Center: R.A. 04:18:23.96 Decl. +55:13:30.6

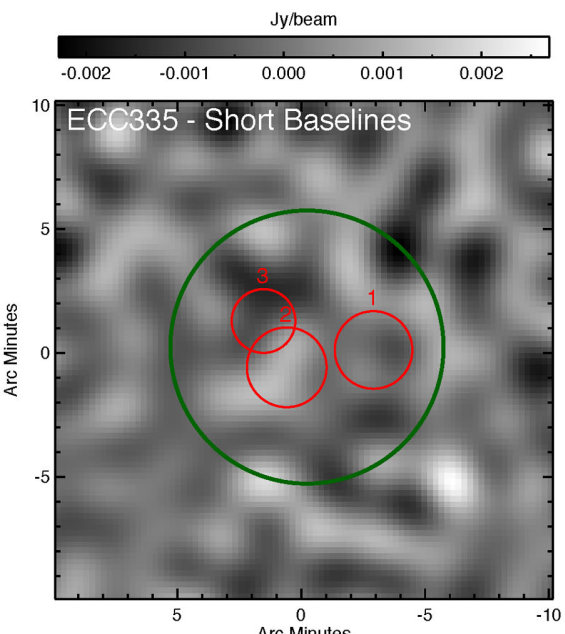

Arc Minutes
Center: R.A. 04:19:11.28 Decl. +55:14:44.4

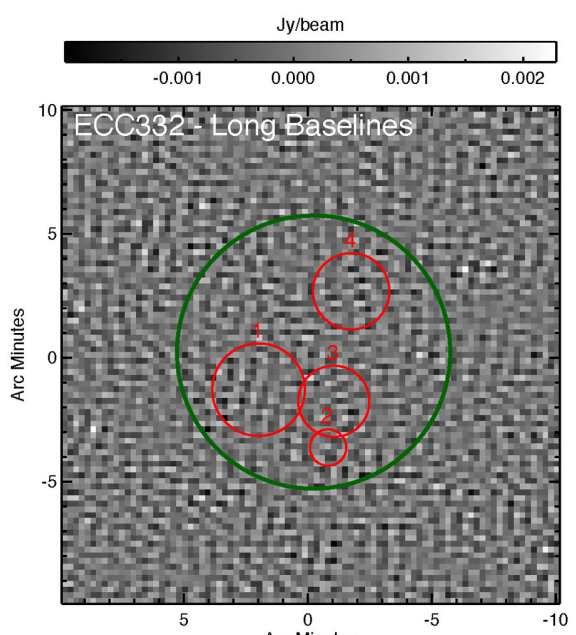

Arc Minutes
Center: R.A. 04:17:09.10 Decl. +55:17:39.4
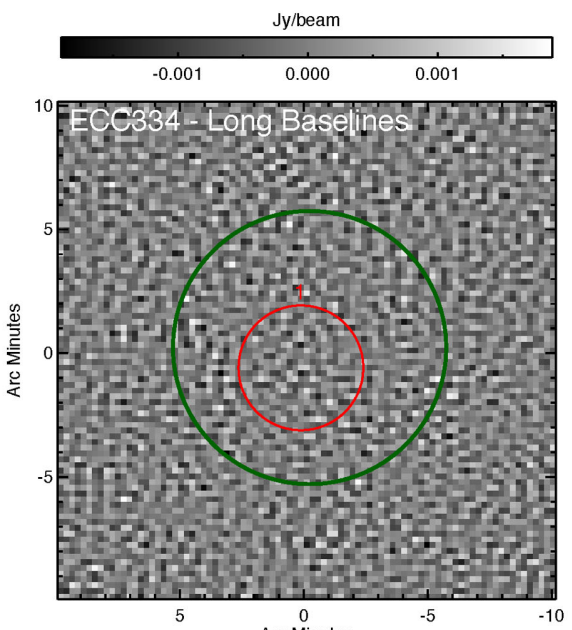

Arc Minutes
Center: R.A. 04:18:23.96 Decl. +55:13:30.6
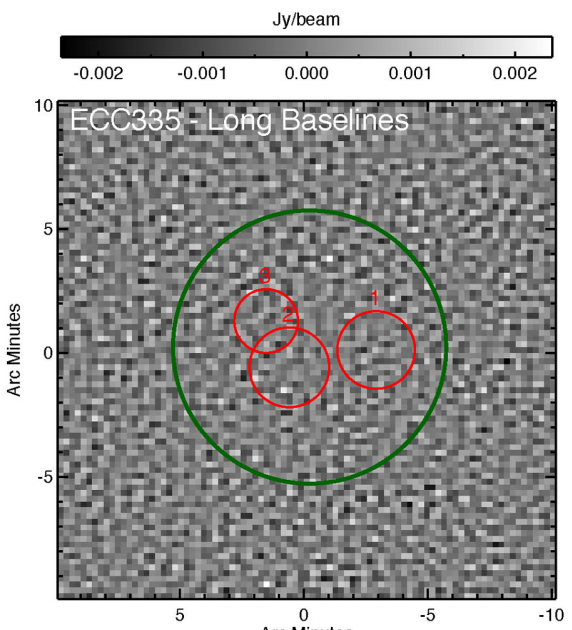

Center: R.A. 04:19:11.28 Decl. +55:14:44.4

Figure A1 - continued 


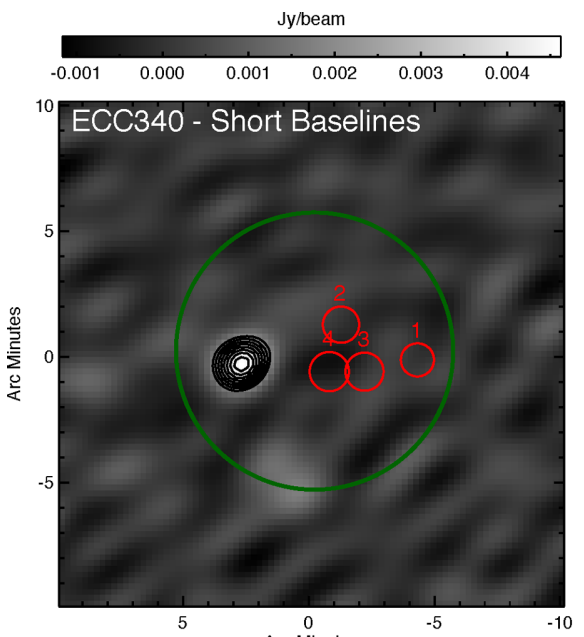

Arc Minutes
Center: R.A. 04:29:56.29 Decl. $+54: 14: 51.7$

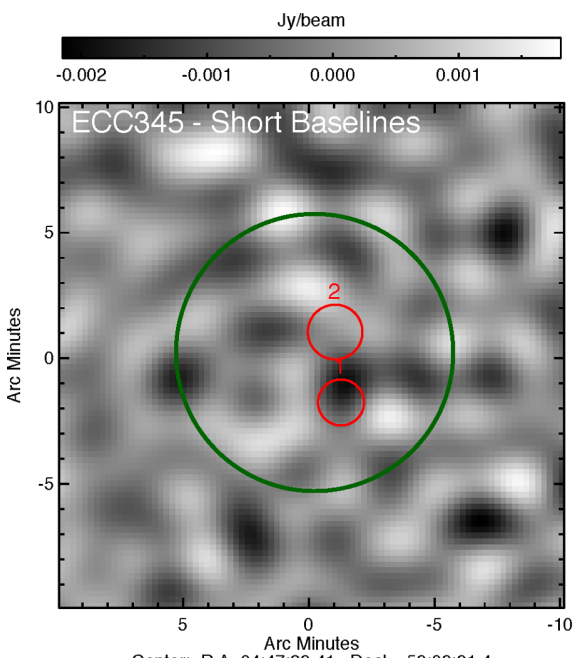

Center: R.A. 04:47:23.41 Decl. +53:03:31.4
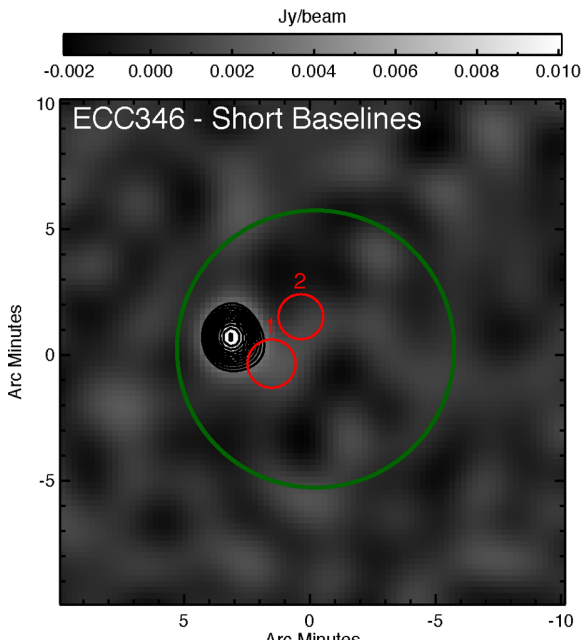

Arc Minutes
Center: R.A. 04:47:57.83 Decl. +53:07:51.2

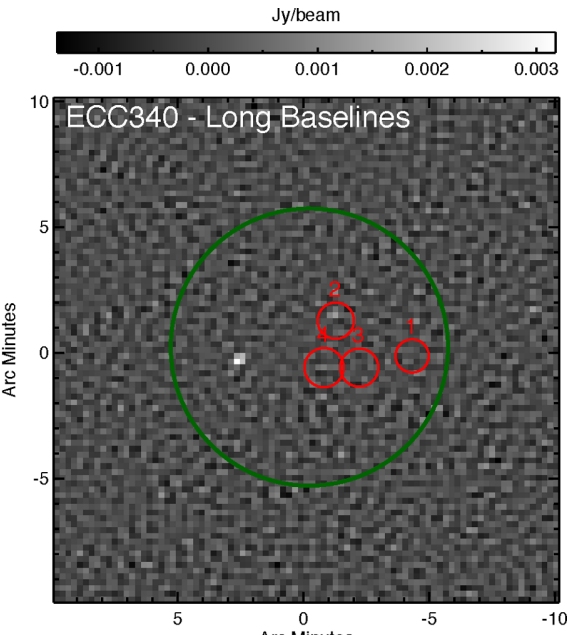

Arc Minutes
Center: R.A. 04:29:56.29 Decl. +54:14:51.7

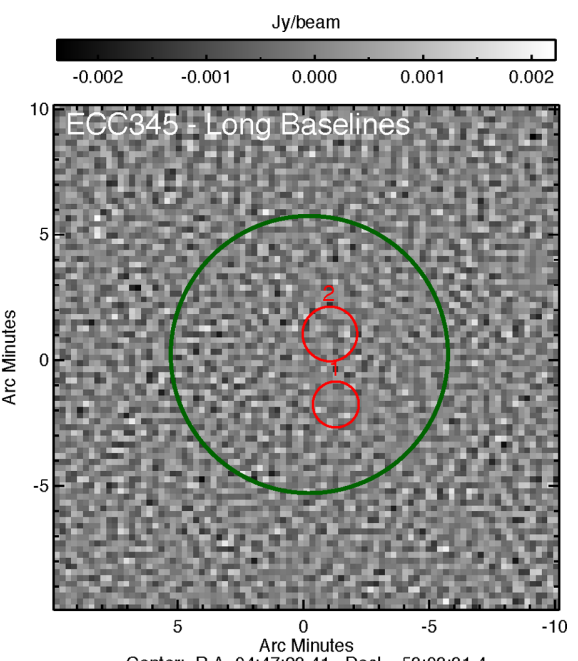

Center: R.A. 04:47:23.41 Decl. +53:03:31.4
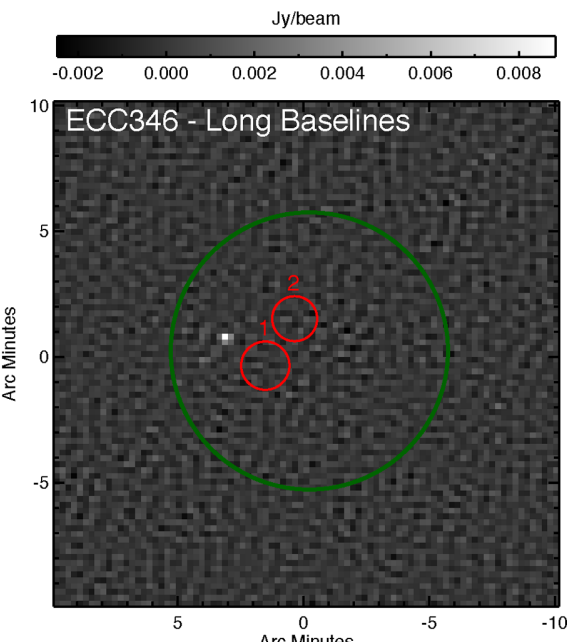

Arc Minutes
Center: R.A. 04:47:57.83 Decl. +53:07:51.2

Figure A1 - continued 

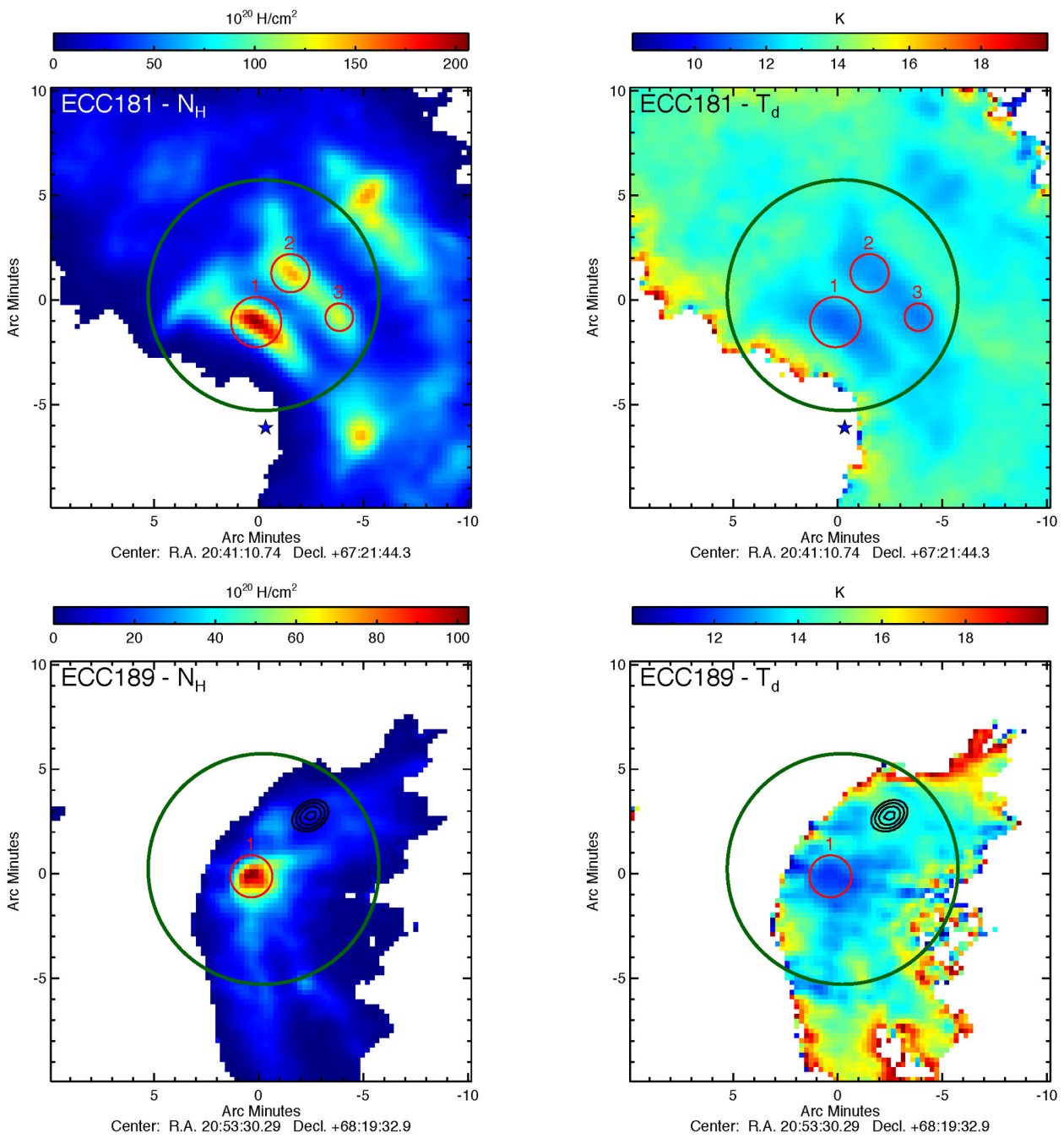

Center: R.A. 20:53:30.29 Decl. $+68: 19: 32.9$
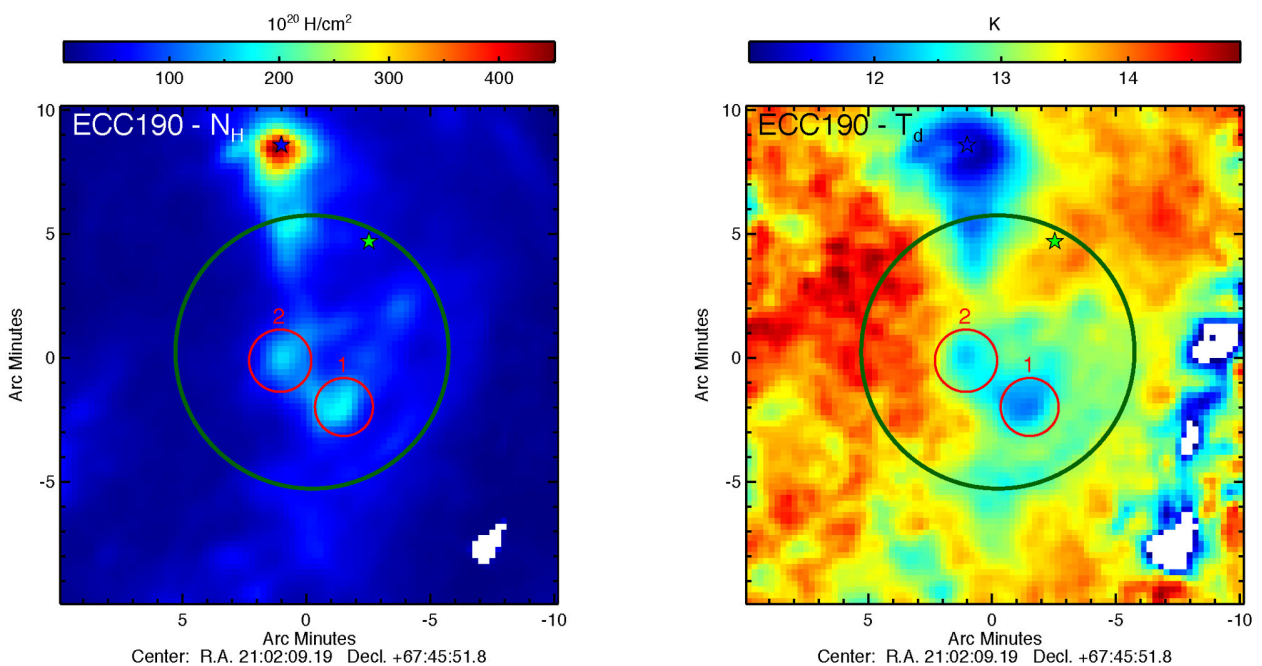

Figure B1. $N_{\mathrm{H}}$ (left) and $T_{\mathrm{d}}$ (right) maps for all 15 cold clumps. The location of the cores are numbered and identified by red circles, while class I and class II YSO candidates are labelled as blue and green stars, respectively. CARMA short-baseline data contours, starting at $5 \sigma$ and increasing linearly, are overplotted (except for ECC191 where the contours increase in steps of 10 $\sigma$ ) along with the CARMA 11 arcmin primary beam (green circle). 

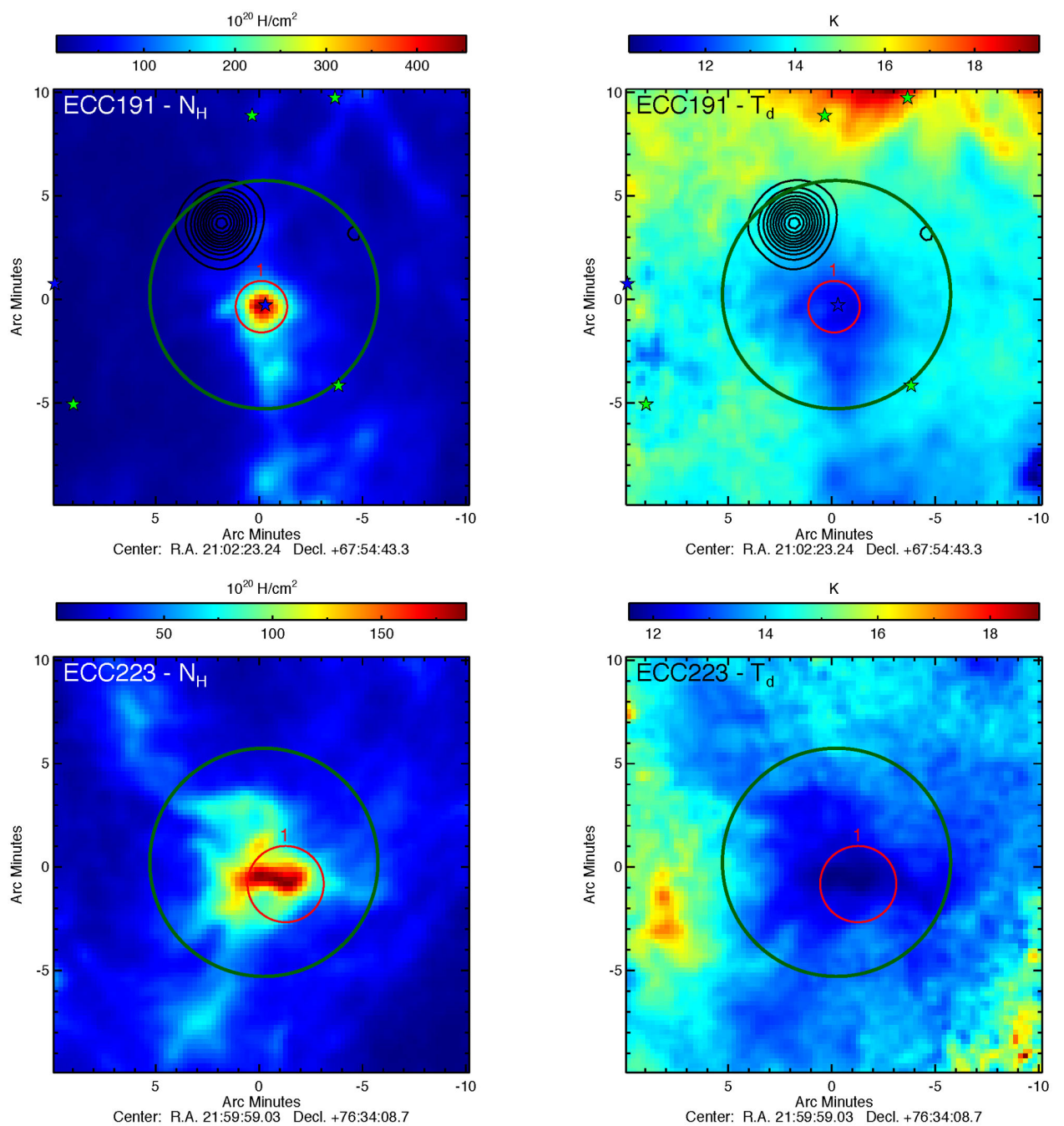

Center: R.A. 21:59:59.03 Decl. +76:34:08.7
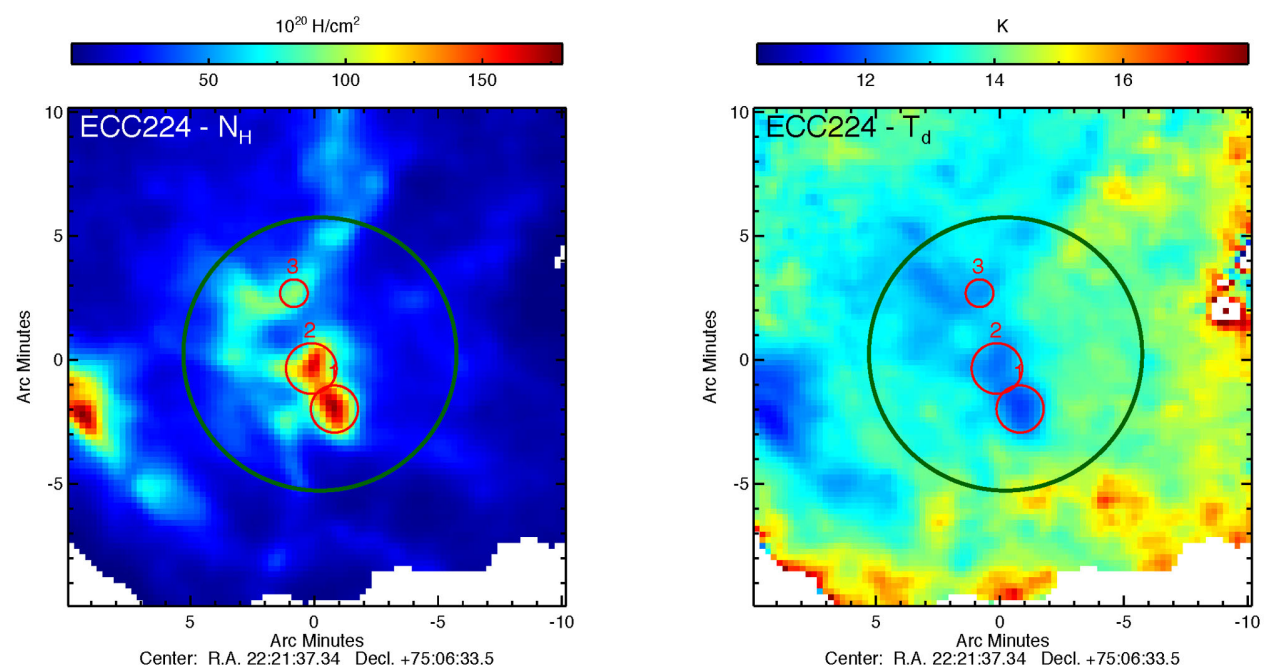

Figure B1 - continued 


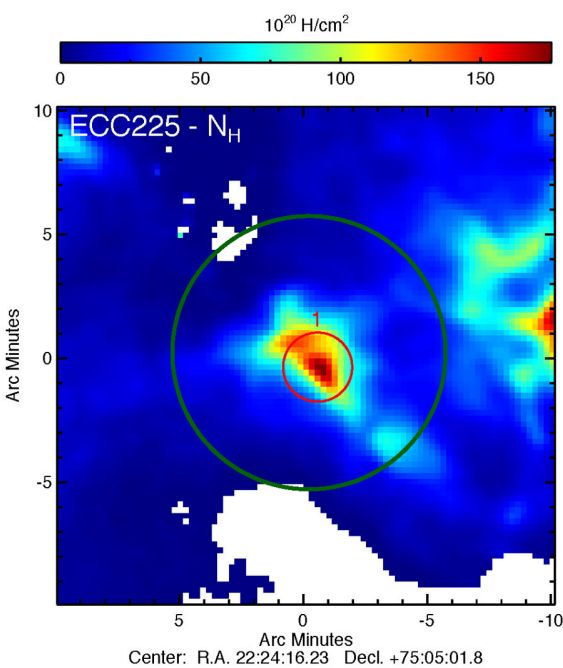

Arc Minutes
Center: R.A. 22:24:16.23 Decl. $+75: 05: 01.8$

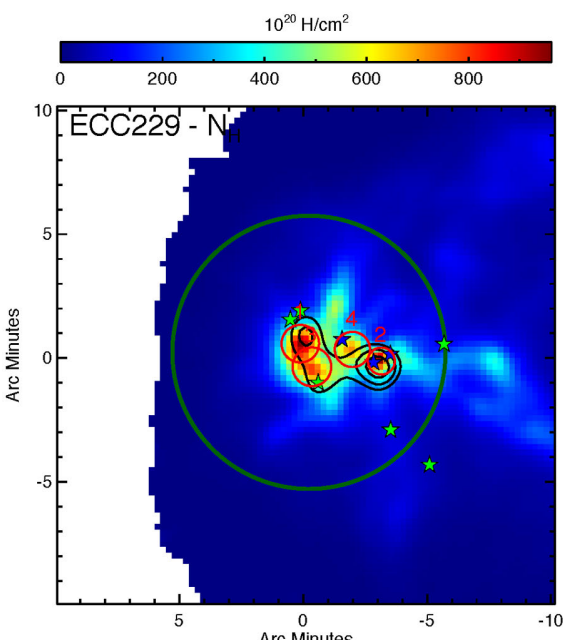

Arc Minutes
Center:

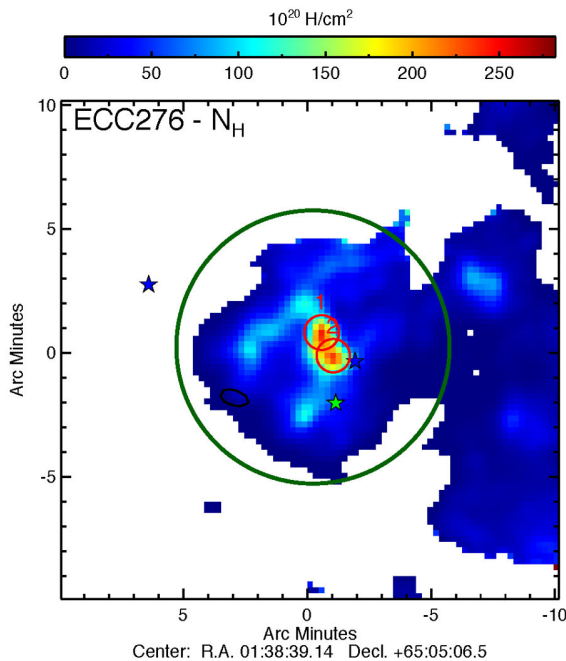

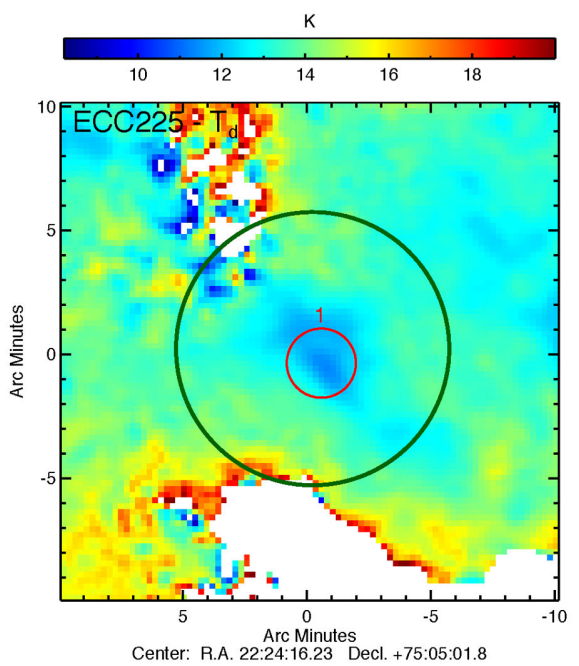

Center: R.A. 22:24:16.23 Decl. +75:05:01.8
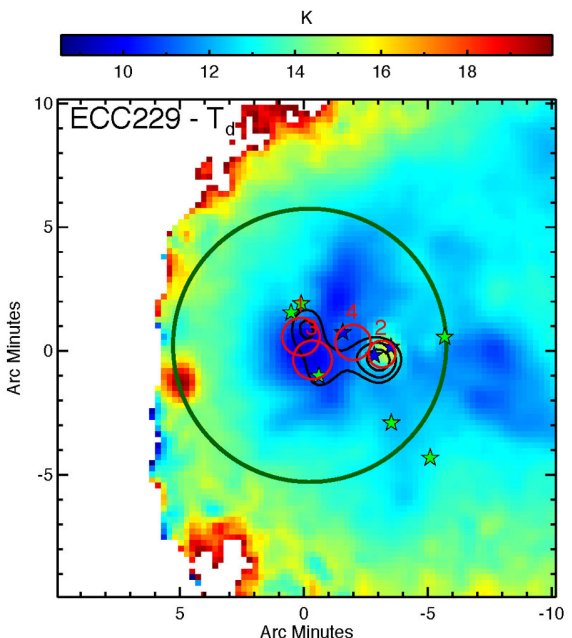

Arc Minutes
Center: R.A. 22:39:35.57 Decl. +75:11:34.0

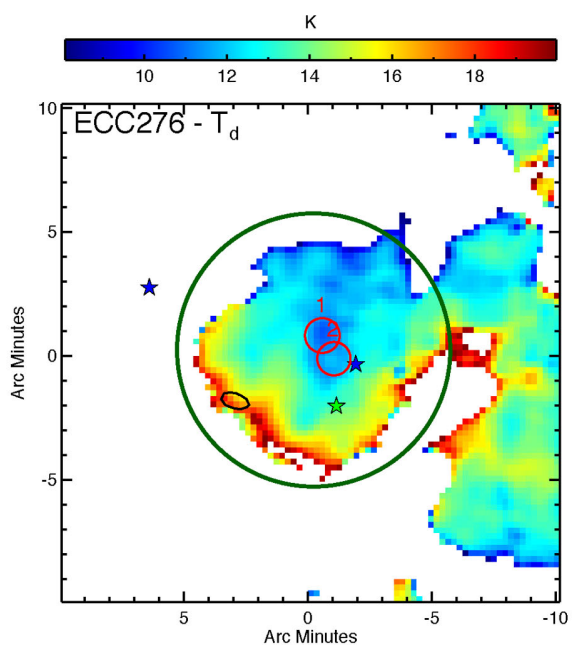

Center: R.A. 01:38:39.14 Decl. +65:05:06.5

Figure B1 - continued 

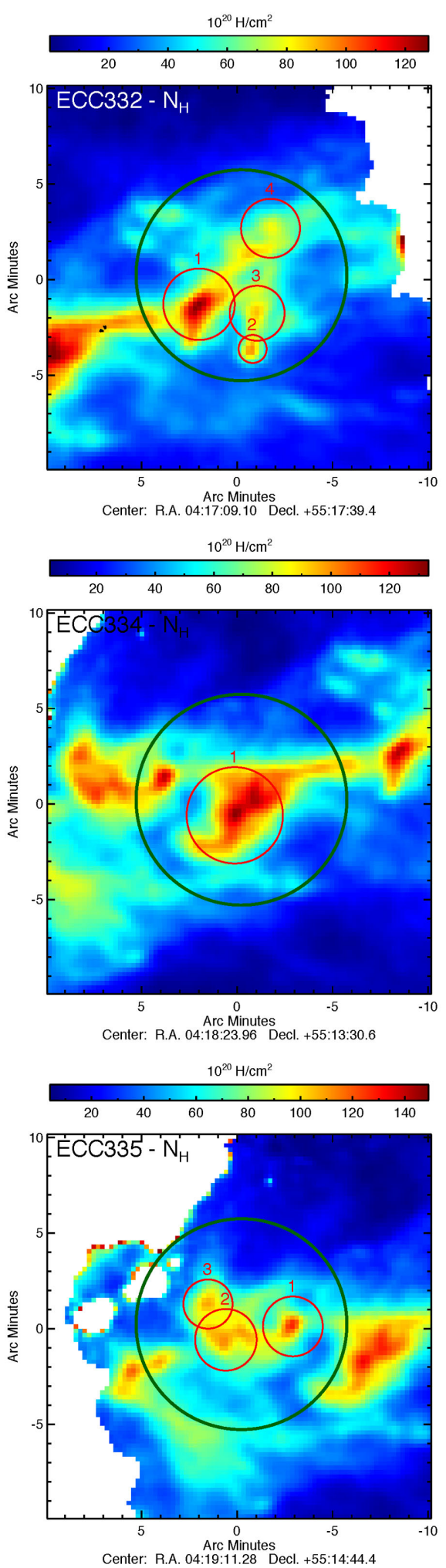
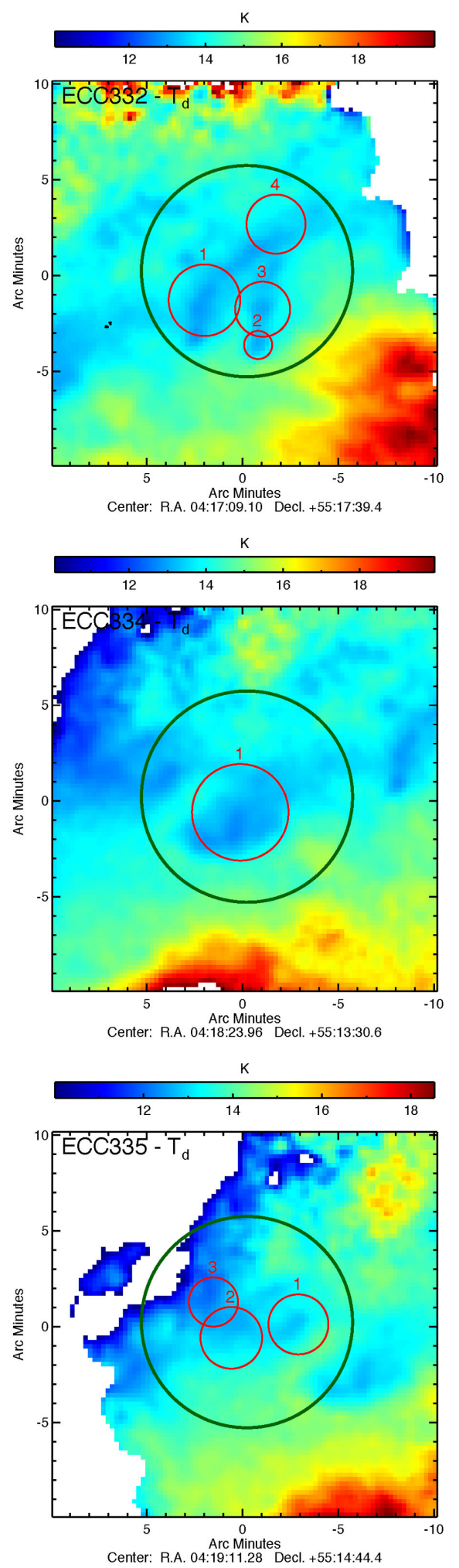

Figure B1 - continued 

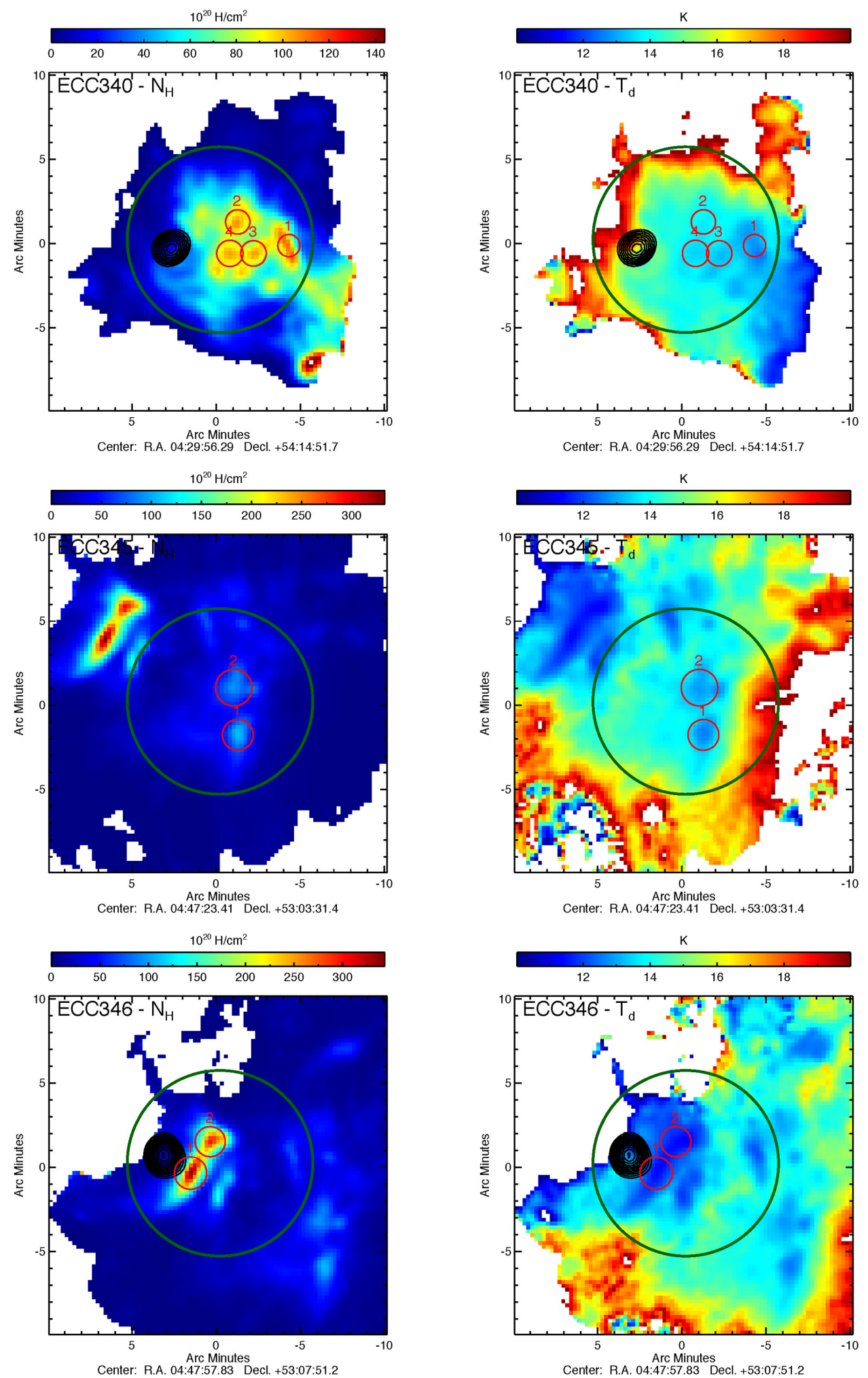

Figure B1 - continued

This paper has been typeset from a $\mathrm{T}_{\mathrm{E}} \mathrm{X} / \mathrm{L} \mathrm{T}_{\mathrm{E}} \mathrm{X}$ file prepared by the author. 